\title{
Turbulent Plumes with Internal Generation of Buoyancy by Chemical Reaction
}

\author{
A. N. Campbell" and S. S. S. Cardoso \\ Department of Chemical Engineering and Biotechnology, University of Cambridge, \\ Pembroke Street, Cambridge CB2 3RA, UK.
}

* Corresponding Author. Fax. +44 1223334796

E-mail address: anc31@cam.ac.uk 


\section{Abstract}

Turbulent plumes, which are seen in a wide number of industrial and natural flows, have been extensively studied; however, very little attention has been paid to plumes which have an internal mechanism for changing buoyancy. Such plumes arise in e.g. industrial chimneys, where species can react and change the density of the plume material. These plumes with chemical reaction are the focus of this study. An integral model describing the behaviour of a plume undergoing a second-order chemical reaction between a component in the plume (A) and a component in the surrounding fluid (B), which alters the buoyancy flux, is considered. The behaviour of a reactive plume is shown to depend on four dimensionless groups: the volume and momentum fluxes at the source, the parameter $\varepsilon$ which indicates the additional buoyancy flux generated by the reaction, and $\gamma$ which is a dimensionless rate of depletion of species B. Additionally, approximate analytical solutions are sought for a reactive plume rising from a point source of buoyancy when species B is in great excess. These analytical results show excellent agreement with numerical simulations. It is also shown that the behaviour of a reactive plume in the far field is equivalent to an inert plume issuing from a virtual source downstream of the real source and the dependence of the location of the virtual source on $\varepsilon$ and $\gamma$ is discussed. The effects of varying the volume flux at the source and the Morton source parameter $\Gamma_{0}$ are further investigated by solving the full governing equations numerically. These solutions indicate that $\varepsilon$ is important in determining the buoyancy generated by the reaction, and the length scale over which this reaction occurs depends on $\gamma$ when $\gamma>1$. It is also shown that when the dimensionless buoyancy $\varepsilon<-1$, the reaction can cause the plume to collapse. 


\section{Introduction}

Turbulent plumes are encountered in a wide variety of natural and industrial flows. Important examples linked to industrial activity are smoke plumes from chimneys or oil fires, effluents from submerged pollution outlets, or even the extreme case of a nuclear accident, while naturally occurring plumes arise at seafloor hydrothermal vents, in convection in clouds, and in explosive volcanic eruptions. These flows range over orders of magnitude in size, but their dynamics are similar.

As a turbulent plume rises above a localised source of buoyancy, it entrains ambient fluid and expands radially. Morton, Taylor and Turner (1956) proposed the most successful macroscopic quantitative description of entrainment, by defining the classical entrainment coefficient:

$\alpha=u_{e} / w$,

where $u_{e}$ is the entrainment velocity at the edge of the plume and $w$ is a measure of the vertical velocity in the plume at the height of interest. The buoyancy flux in the plume depends on the buoyancy released at the source and on vertical density changes in the surrounding environment. The interactions between the plume and its surroundings, and the evolution of buoyancy in the plume induced by the environmental density stratification have been studied extensively for more than fifty years (List, 1982).

However, there are many examples of plumes which have internal mechanisms for generating buoyancy, in addition to the buoyancy released at the source and any possible changes induced by external stratification. In oil refinery flares, in fires and other chemical plumes, the ongoing chemical reactions generate large fluxes of buoyancy within the plume. For example, Hanna, Chang and Zhang (1997) considered the accidental release of uranium hexafluoride from pipes or vessels. Depending on the physical conditions, the released $\mathrm{UF}_{6}$ can initially form a dense mixture of gas and solid particles. The particles sublime, thus absorbing heat $\left(\Delta H_{S}=50 \mathrm{~kJ} \mathrm{~mol}^{-1}\right)$ making the plume more dense. The $\mathrm{UF}_{6}$ can also react with water vapour to form $\mathrm{HF}\left(\Delta H_{r x n}=-58.6 \mathrm{~kJ} \mathrm{~mol}^{-1}\right)$ which reacts further with water $\left(\Delta H_{r x n}\right.$ $\left.=-26.2 \mathrm{~kJ} \mathrm{~mol}^{-1}\right)$. There is therefore a combination of reactions that can cause the plume to switch from being denser than air to less dense. In volcanic plumes, hot clasts heat up entrained air generating the buoyancy responsible for the large heights of rise in the atmosphere, and in clouds and in discharges from power plant chimneys, transitions of phase can induce important density changes. These types of plumes, with internally-produced buoyancy changes, have been the focus of comparatively few studies. 
Previous work on plumes or jets with internal buoyancy generation has been primarily concerned with understanding the behaviour of cumulus clouds, where latent heat effects can cause changes in buoyancy, and with volcanic eruption columns. In analogy with cumulus clouds, the behaviour of buoyant jets heated volumetrically by passing an electric current through them at some distance above the source, has been studied experimentally by Bhat, Narasimha and Arakeri (1989). Their initial experiments on turbulent jets indicated that the rate of radial spread of the jet was reduced in the presence of significant volumetric heating. Further studies (e.g. Bhat and Narasimha, 1996; Venkatakrishnan, Bhat and Narasimha, 1996; Agrawal and Prasad, 2004; Agrawal, Sreenivas and Prasad, 2004) confirmed the reduction in plume width with heat addition, and observed that this effect was coupled with an acceleration of the fluid and with a disruption of the large scale eddies in the flow. These studies also showed that just downstream of the zone of heat addition, the rate of entrainment increased, whereas farther downstream, the rate of entrainment was slightly lower.

The different behaviours exhibited by volcanic eruption columns were studied experimentally by Woods and Caulfield (1992). Their experiments were conducted by injecting a buoyant mixture of methanol and ethylene glycol (MEG) downwards into a tank of fresh water. When the MEG mixes with water, it can become denser than water and form a convecting plume. This system allowed the study of the conditions for which the plume is stable and for which it collapses. Woods and Caulfield (1992) developed a simple model to predict collapse. Their modelling was later extended (Caulfield and Woods, 1995) for a general plume where the two fluids exhibit non-linear mixing. More recently, a similar system using ethanol and ethylene glycol (EEG) was used by Kaminski, Tait and Carazzo (2005) to investigate the effects of buoyancy generation on entrainment. They derived an expression for the entrainment coefficient $\alpha$ based on the local Richardson number $(R i)$ and a number of integral parameters of the plume.

In addition to the experiments described above, there have been a limited number of attempts at modelling plumes or jets with internal generation of buoyancy. Hunt and Kaye (2005) considered the effect of buoyancy generation on so-called 'lazy plumes', i.e. plumes with a deficit of momentum at the source compared with a pure plume with the same source buoyancy flux. The model they developed was based on that of Morton et al. (1956) and assumed Gaussian profiles within the plume. Hunt and Kaye (2005) considered a case where the buoyancy flux increased linearly with height and were able to define a length scale at which the buoyancy flux generated during ascent became equal to that released at the source. 
The model showed that a plume with internal generation of buoyancy was narrower than the corresponding non-reactive plume.

A similar analysis to that described by Hunt and Kaye (2005) was used by Ishimine (2007) to investigate the behaviour of eruption columns with internal buoyancy generation. Ishimine (2007) considered plumes with a linear variation of buoyancy flux with height as well as plumes in which the buoyancy flux changed with distance above the source $z$ according to

$B=B_{\infty}(1-\exp [-z / L])$.

Here, the buoyancy flux increases from zero to a constant value $B_{\infty}$ far above the source. Ishimine (2007) investigated the behaviour of plumes with such variations in buoyancy flux in both stratified and uniform environments. As in Hunt and Kaye (2005), no detailed mechanisms to generate such a variation of buoyancy flux were discussed. It should also be noted that Ishimine (2007) did not use the classical entrainment hypothesis described above (equation (1.1)), but instead assumed that the plume radius increased linearly with height as discussed by e.g. Batchelor (1954) and Priestley and Ball (1955). Both Ishimine (2007) and Hunt and Kaye (2005) showed that for a plume with a buoyancy flux that increases linearly with height, the velocity of the plume was independent of the height above the source in the far-field.

Diez and Dahm (2007) developed a new approach to model the behaviour of a turbulent buoyant jet flame. In such a flame, the rate of reaction is very fast, hence the progress of the reaction, and consequently buoyancy generation are limited by mixing. The model utilised an equivalence principle, developed in previous studies (Tacina and Dahm, 2000; Dahm, 2005) of different shear flows undergoing reaction controlled by mixing. The method involves the superposition of two non-reactive plumes to describe the behaviour in the reactive case. The authors developed an integral model for a non-reacting plume. As with Ishimine (2007), they did not use the entrainment hypothesis, but instead relied on the empirical observation that the spreading coefficient in jets and plumes is approximately the same. In addition, when modelling the change of buoyancy flux with height, they assume that the rate of reaction is one third of the rate of entrainment (characterised by the rate of change of mass with height), again based on some empirical measurements. Diez and Dahm (2007) showed that their method agreed well with measured flame lengths and velocities in buoyant jet flames.

Recently, Conroy and Llewellyn Smith (2008) formulated a plume model in which the effects of an exothermic or endothermic reaction on the plume dynamics were considered. 
This work extended their previous study (Conroy, Llewellyn Smith and Caulfield, 2005) of a plume undergoing a chemical reaction which had no effect on the buoyancy flux. Conroy and Llewellyn Smith (2008) inserted an expression for the variation of density in a flowing, reactive system, as derived by e.g. Clarke and McChesney (1964) and Williams (1985), into the top-hat reactive plume model of Conroy et al. (2005), in which the Boussinesq approximation is made, and also into a model for non-Boussinesq plumes. It was shown that the effect of a chemical reaction on the plume density could be summarised by a parameter, $\sigma$, which combined the thermodynamic effects of the reaction on the density with the local rate of reaction. In combustion science, where $\sigma$ is often used in the modelling of flames, this parameter is taken to represent the rate of heat liberation by the chemical reaction, but it can also include density changes caused by the changing composition of the reactive mixture. Conroy and Llewellyn Smith (2008) then presented numerical simulations for a Boussinesq plume, with and without stratification in the environment, for perfect gasses undergoing the second-order reaction $S_{1}+S_{2} \rightarrow S_{3}$. Even in the absence of any heat of reaction, such a reaction would always cause the plume to collapse (in the absence of any additional source of buoyancy) because of the production of a heavier molecule from two lighter ones. Additionally, they presented an approximate analytical solution in the absence of stratification for the limiting case where both the rate of reaction and the heat of reaction are very large. Finally, numerical simulations of a non-Boussinesq plume without external stratification were discussed.

There are, however, some problems with Conroy and Llewellyn Smith's (2008) analysis. The numerical solutions for the Boussinesq plume in the absence of stratification are presented for different values of a dimensionless parameter (their $\lambda_{3}$ ), which they consider to be a dimensionless rate of reaction. However, the scheme used to make the governing equations dimensionless indicates that this parameter is in fact given by $(2 \alpha)^{2 / 3} M_{3} / M_{1}$, where $M_{i}$ represents the molar mass of species $i$. Given that throughout the paper it is assumed that $M_{1}=M_{2}=1$ (and hence via the reaction stoichiometry above $M_{3}=2$ ) the numerical results presented in figures 2 and 3 of Conroy and Llewellyn Smith (2008), which were produced by varying $\lambda_{3}$, are in fact unphysical. The approximate analytical solution for a Boussinesq plume undergoing a very fast reaction with a large heat of reaction in an unstratified environment also merits discussion. Physically, this limit corresponds to a mixing controlled reaction, as discussed by Diez and Dahm (2007). Given the assumption of a large heat of reaction, it is questionable whether the Boussinesq approximation is appropriate in 
such a limit, especially for a perfect gas plume as discussed by Conroy and Llewellyn Smith (2008). The authors assumed that the rate constant $k \gg>1$ and hence $\lambda_{2}>>1$; however, the parameter $\lambda_{2}=(2 \alpha)^{2 / 3} M_{2} / M_{1}$ is independent of $k$. It is also assumed that the rate of change of the flux of species B with height above the source is $\sim 0$ due to the rapid reaction and thus the two terms in the equation governing $\mathrm{d} F_{2} / \mathrm{d} z$ could be balanced. This balance was subsequently used to substitute for the reaction term in the buoyancy flux equation, allowing the equation to be written purely in terms of the momentum flux. This assumption requires that $\lambda_{2} F_{2} \sim O(1)$, which is not the case for $k>>1$. The equations governing the volume, momentum and buoyancy fluxes were then solved, whilst neglecting the fact that species $\mathrm{A}$ in the plume would be depleted and thereby ignoring the fact that the reaction would cease when A is exhausted. The analytical solutions therefore are only valid for a small height above the source and yield no information about the behaviour in the far-field, or the total additional buoyancy flux generated by the chemical reaction. Finally, for the simulations of the Boussinesq plume in the presence of external stratification the mass fraction of the species in the environment $\left(R_{20}\right)$ at the source of the plume is defined as being 1 . The stratification of this species is governed by a relation of the form $R_{2}=R_{20}\left(z / z_{s}\right)^{\gamma}$. The simulations presented in figure 5 of Conroy and Llewellyn Smith (2008) show the parameter $\gamma$ varying between -2 and 4 . The positive values of $\gamma$ represent cases where the mass fraction of species 2 in the environment increases above 1, which is again unphysical.

This work develops a model for a reactive Boussinesq plume in which buoyancy is generated by the reaction in the absence of external stratification. An approximate analytical solution to the governing equations is sought in the limit of plumes which generate a relatively small amount of additional buoyancy. Additionally, a detailed numerical investigation of the effects of varying the key dimensionless parameters is performed.

\section{Theoretical Development}

\subsection{Model Chemistry}

Here, the reaction considered is the general second-order reaction:

$\left|v_{A}\right| S_{A}+\left|v_{B}\right| S_{B} \rightarrow\left|v_{C}\right| S_{C}+\left|v_{D}\right| S_{D}$

where the $S_{i}$ represent the species involved in the reaction, and the corresponding stoichiometric coefficients, $v_{i}$, are defined as being positive for products and negative for reactants. Hence, reaction (2.1) can be written as: 
$\sum_{i} v_{i} S_{i}=0$

Species A is considered to be in the plume whilst species B is in the environment. The enthalpy of reaction is defined as:

$\Delta H_{r x n}=\sum_{i} v_{i} \Delta \underline{H}_{f, i}$

where $\Delta \underline{H}_{f, i}$ is the heat of formation of component $i$. By convention, $\Delta H_{r x n}$ is defined as being negative for an exothermic reaction. The rate of the reaction per unit volume with respect to species $i$ is given by:

$r_{i}=v_{i} k C_{A} C_{B}$,

where $k$ is the rate constant and $C_{i}$ represents the concentration of species $i$. It is assumed throughout that the rate constant $k$ is independent of temperature. This approximation is valid for dilute mixtures because any changes in temperature induced by the reaction are then small.

\subsection{Plume Equations}

The governing conservation equations for the plume can now be written. Following the method proposed by Morton et al. (1956), the time-averaged properties of the plume are considered; the turbulent contributions are neglected. Analysis of a ring-shaped volume of fluid in the plume yields the following equations. Conservation of mass is given by continuity:

$\frac{1}{r} \frac{\partial(r u)}{\partial r}+\frac{\partial w}{\partial z}=0$

where $r$ and $z$ are the radial and vertical coordinates, and $u$ and $w$ are the radial and vertical velocities. Conservation of species $i$ can be written as

$u \frac{\partial C_{i}}{\partial r}+w \frac{\partial C_{i}}{\partial z}=v_{i} k C_{A} C_{B}$,

conservation of vertical momentum is

$\rho_{\infty}\left(u \frac{\partial w}{\partial r}+w \frac{\partial w}{\partial z}\right)=-\frac{\partial p}{\partial z}-\rho g$

where $p$ is the pressure in the plume, $\rho$ is the density and $\rho_{\infty}$ is the density of the environment. The conservation of thermal energy is given by

$u \frac{\partial T}{\partial r}+w \frac{\partial T}{\partial z}=\frac{k C_{A} C_{B}\left(-\Delta H_{r x n}\right)}{\rho_{\infty} c_{p}}$. 
The formulation of these equations assumes that the Boussinesq approximation holds, i.e. the density changes are only important in the final term of equation (2.7).

Within the plume, each quantity is considered to have a Gaussian distribution in the radial direction:

$$
\theta(r, z)=\theta_{\infty}+\theta_{g}(z) \exp \left(-r^{2} / b(z)^{2}\right)
$$

where the subscripts $\infty$ and $g$ denote the property $\theta$ at a large radial position and the centreline of the plume, respectively. This assumption should hold when the rate of reaction is much slower than the rate of mixing due to turbulence. It should be noted that the widths of all the Gaussian profiles have for simplicity been taken to be equal. It is also assumed that the ambient fluid is free from species $\mathrm{A}, \mathrm{C}$ and $\mathrm{D}$ and has zero vertical velocity, hence $C_{A, \infty}=$ $C_{C, \infty}=C_{D, \infty}=w_{\infty}=0$. The distribution used in equation (2.9) also means that the value of $C_{B, g}$ is negative, since the concentration of $\mathrm{B}$ will decrease as the radial position $r$ decreases from the environment to the axis of the plume.

The plume behaviour can be described in terms of the following integrated timeaveraged fluxes:

volume flux: $Q(z)=\int_{0}^{\infty} 2 \pi r w d r=\pi b^{2} w_{g}$,

momentum flux: $M(z)=\int_{0}^{\infty} 2 \pi r w^{2} d r=(1 / 2) \pi b^{2} w_{g}^{2}$,

buoyancy flux: $B(z)=\int_{0}^{\infty} 2 \pi r w g^{\prime} d r$,

flux of species A: $F_{A}(z)=\int_{0}^{\infty} 2 \pi r w C_{A} d r=(1 / 2) \pi b^{2} w_{g} C_{A, g}$,

flux of species B: $F_{B}(z)=\int_{0}^{\infty} 2 \pi r w C_{B} d r=\pi b^{2} w_{g}\left(C_{B, \infty}+(1 / 2) C_{B, g}\right)$,

flux of enthalpy: $H(z)=\int_{0}^{\infty} 2 \pi r w \rho_{\infty} c_{p}\left(T-T_{\infty}\right) d r=(1 / 2) \rho_{\infty} c_{p} \pi b^{2} w_{g} T_{g}$.

Here, $g^{\prime} \equiv g\left(\rho_{\infty}-\rho\right) / \rho_{\infty}$ is the reduced gravity. The fluxes of C and D have not been included because they are related to the flux of A via the stoichiometry of reaction (2.1). Equation (2.15) for the enthalpy flux uses $T_{\infty}$ as the datum temperature for enthalpy and assumes that the specific heat of the fluid, $c_{p}$, is independent of temperature. In order to 
determine the integral in equation (2.12), the density of the plume material must be evaluated. For dilute solutions, the density may be written as:

$\rho=\rho_{\text {sol }}\left(1+\sum K_{i} C_{i}-\beta\left(T-T_{\infty}\right)\right)$

where $\rho_{\text {sol }}$ is the density of the solvent and $\beta$ denotes the coefficient of thermal expansion. This expression is simply a Taylor series expansion of density about a pure solvent. Such expressions have been used often in the analysis of plumes (e.g. Morton et al., 1956). The constants $K_{i}$ are therefore defined as $\left(1 / \rho_{\text {sol }}\right)\left(\partial \rho / \partial C_{i}\right)$ and can easily be determined from experiments. Substituting equation (2.16) into equation (2.12) yields:

$$
B(z)=\int_{0}^{\infty} 2 \pi r w g^{\prime} d r=\frac{\pi b^{2} w_{g} g}{2\left(1+K_{B} C_{B, \infty}\right)}\left(\beta T_{g}-\sum K_{i} C_{i, g}\right) .
$$

Using equations (2.5) - (2.8), it may be shown that the variation of the fluxes of volume, momentum, buoyancy and species A and B, with height in the plume are given by:

$$
\begin{aligned}
& \frac{d Q}{d z}=(8 \pi)^{1 / 2} \alpha M^{1 / 2} \\
& \frac{d M}{d z}=\frac{B Q}{M} \\
& \frac{d B}{d z}=\frac{k F_{A} F_{B}}{M} \frac{g \rho_{\text {sol }}}{\rho_{\infty}}\left(\frac{\beta\left(-\sum v_{i} \Delta \underline{H}_{f, i}\right)}{\rho_{\infty} c_{P}}-\sum v_{i} K_{i}\right) \\
& \frac{d F_{A}}{d z}=-\left|v_{A}\right| \frac{k F_{A} F_{B}}{M} \\
& \frac{d F_{B}}{d z}=(8 \pi)^{1 / 2} \alpha M^{1 / 2} C_{B, \infty}-\left|v_{B}\right| \frac{k F_{A} F_{B}}{M}
\end{aligned}
$$

Equations (2.18) - (2.22) are sufficient to describe the dynamics of the plume, because the effects of varying the fluxes of $\mathrm{C}$ and $\mathrm{D}$, and the effect of varying the temperature, are accounted for in equation (2.20). A further equation for the variation of $H$ would be required if the rate constant for the chemical reaction were a function of temperature. Equation (2.20) can be re-written as:

$$
\frac{d B}{d z}=\frac{k F_{A} F_{B} g_{r x n}^{\prime}}{M}
$$

where $g_{r x n}^{\prime}$ is defined as

$$
g_{r x n}^{\prime} \equiv \frac{g \rho_{s o l}}{\rho_{\infty}}\left(\frac{\beta\left(-\sum v_{i} \Delta \underline{H}_{f, i}\right)}{\rho_{\infty} c_{P}}-\sum v_{i} K_{i}\right) \text {. }
$$


Thus, in contrast to the parameter $\sigma$ derived by e.g. Clarke and McChesney (1964), which varies locally throughout the plume, the effect of chemical reaction on the buoyancy flux is characterised by a constant $g_{r x n}^{\prime}$ which can easily be defined for a given reaction. It can also be shown that the parameter $g_{r x n}^{\prime}$ is consistent with the expression for the effect of chemical reaction on density derived in Clarke and McChesney (1964). There are clearly two contributions to $g_{r x n}^{\prime}$, one that accounts for thermal effects and another accounting for compositional changes. When $g_{r x n}^{\prime}$ is positive, the reaction causes the buoyancy flux in the plume to increase.

Equations (2.20) and (2.23) could be straightforwardly modified to include the effects of external stratification by the inclusion of the familiar $\left(-N^{2} Q\right)$ term on the right hand side, where $N^{2}$ is the buoyancy frequency, which in this case would be defined as:

$$
N^{2}=-\frac{g}{\rho_{\infty}} \frac{d \rho_{\infty}}{d z}=\beta g \frac{d T_{\infty}}{d z}-K_{B} \frac{\rho_{\text {sol }} g}{\rho_{\infty, 0}} \frac{d C_{B, \infty}}{d z}-K_{I} \frac{\rho_{\text {sol }} g}{\rho_{\infty, 0}} \frac{d C_{I, \infty}}{d z}
$$

where $\rho_{\infty, 0}$ is the density of the environment at the source and the subscript $I$ relates to any inert chemical species which contribute to the density stratification. Considering equation (2.23) reveals that a reactive plume which generates buoyancy is therefore equivalent to an inert plume rising in a stratified environment with

$$
N^{2}=-\frac{k F_{A} F_{B} g_{r x n}^{\prime}}{Q M}
$$

A knowledge of the fluxes in a reactive plume would allow the equivalent stratification to be found for an inert plume. In a reactive plume, the flux of A will be reduced to zero by the chemical reaction. Equation (2.26) therefore implies that for the equivalent inert plume, the density should vary for a finite height above the source before it reaches a constant value in the far-field. It is also clear from equation (2.26) that when the chemical reaction generates buoyancy (i.e. $g_{r x n}^{\prime}>0$ ), then the equivalent stratification would be unstable (with density increasing with height) and vice versa.

Batchelor (1954) produced similarity solutions for plumes rising in unstably stratified environments. The analysis assumed a power-law variation in buoyancy frequency of the form $N^{2}=-N_{0}^{2} z^{p}$ where $N_{0}$ is constant and $p>-8 / 3$. Whilst the variation in equivalent buoyancy frequency described in equation (2.26) is more complex than the power-law variation considered by Batchelor (1954), some qualitative comparison can be made. The equivalent buoyancy frequency for a reactive system will reach zero at a finite height above 
the source, the power-law buoyancy frequency will approach zero asymptotically if $p<0$. The similarity solutions produced by Batchelor (1954) show that plumes rising into unstably stratified environments with $-8 / 3<p<0$ will be narrower than those rising in a uniform environment. Interestingly, if $-2<p<0$ the similarity solutions indicate that the plume velocity will actually increase with height above the source.

As well as drawing an analogy between the effects of buoyancy generation by chemical reaction and external stratification on the behaviour of a plume, it is also possible to compare the effects of chemical reaction to those of a time-dependent source of buoyancy, as considered by Scase et al. (2006). They examined a plume in which the buoyancy flux was reduced from an initial constant value to a final constant value. When viewed at a particular time, the plume would have a region of high buoyancy flux far from the source (corresponding to the initial buoyancy flux), a region of low buoyancy flux (the final value of buoyancy flux) close to the source and some transition region. The buoyancy flux is therefore varying with height, much like if a chemical reaction was occurring or there was an unstable density distribution in the environment. Scase et al. (2006) found that in the regions of constant buoyancy flux close to, and very far from the source, the plume behaved according to the similarity solutions of Morton et al. (1956), whereas in the transition region, the velocity increased in proportion with distance from the source and the plume width would first decrease with height, before tending back to the width predicted by Morton et al. (1956).

\subsection{Dimensionless Plume Equations}

The governing equations $(2.18)-(2.22)$ can be made dimensionless through the introduction of the variables:

$$
\hat{Q}=Q / Q_{S} ; \hat{M}=M / M_{S} ; \hat{B}=B / B_{0} ; \hat{F}_{A}=F_{A} / F_{A, 0} ; \hat{F}_{B}=F_{B} / F_{B, S} ; \hat{z}=z / L .
$$

The buoyancy flux at the source $B_{0}$ is a natural scale for the buoyancy flux. Similarly, the flux of species A at the source is also a natural scale. It may be recalled that in the case of an inert plume issuing from a point source of buoyancy, no length scale can be derived (Morton et al., 1956). However, when the plume fluid does react with the fluid in the environment, the following scales may be defined:

$$
\begin{aligned}
& L \sim \frac{B_{0}^{1 / 4}}{2^{3 / 4} \pi^{1 / 4} \alpha^{1 / 2}\left(\left|v_{A}\right| k C_{B, \infty}\right)^{3 / 4}}, \\
& Q_{S} \sim \frac{2^{3 / 4} \pi^{1 / 4} \alpha^{1 / 2} B_{0}^{3 / 4}}{\left(\left|v_{A}\right| k C_{B, \infty}\right)^{5 / 4}},
\end{aligned}
$$




$$
\begin{aligned}
& M_{S} \sim \frac{B_{0}}{\left|v_{A}\right| k C_{B, \infty}} \text { and } \\
& F_{B, S} \sim \frac{2^{3 / 4} \pi^{1 / 4} \alpha^{1 / 2} B_{0}^{3 / 4} C_{B, \infty}}{\left(\left|v_{A}\right| k C_{B, \infty}\right)^{5 / 4}}=Q_{S} C_{B, \infty} .
\end{aligned}
$$

Unlike the non-reactive case, a natural length scale does emerge. Here, $L$ gives an indication of the height above the source over which the plume has generated significant buoyancy through reaction with the environment, compared to that issuing at the source. The other scales were defined primarily for mathematical convenience; however, they do represent characteristic values of the fluxes at a distance $L$ from the source. The length scale in equation (2.28) is similar to that presented by Conroy and Llewellyn Smith (2008), although it should be noted that the expression for $H_{C}$ they presented does not, in fact, have dimensions of length. Instead $H_{C}$ has dimensions $\mathrm{m} \mathrm{mol}^{3 / 2} \mathrm{~kg}^{-3 / 2}$. It is also interesting to compare the form of the length scale in equation (2.28) with the scale for maximum rise height of a plume in a stably stratified environment derived by Morton et al. (1956) as:

$L \sim \alpha^{-1 / 2} B_{0}^{1 / 4} N^{-3 / 4}$,

thus, as discussed above, an analogy can clearly be drawn between the effects of the chemical reaction and external stratification and will be discussed further below. Using the scales in equations $(2.28)-(2.31)$, the governing equations become:

$$
\begin{aligned}
& \frac{d \hat{Q}}{d \hat{z}}=\hat{M}^{1 / 2}, \\
& \frac{d \hat{M}}{d \hat{z}}=\frac{\hat{B} \hat{Q}}{\hat{M}}, \\
& \frac{d \hat{B}}{d \hat{z}}=\frac{F_{A, 0} g_{r x n}^{\prime}}{\left|v_{A}\right| B_{0}} \frac{\hat{F}_{A} \hat{F}_{B}}{\hat{M}}=\varepsilon \frac{\hat{F}_{A} \hat{F}_{B}}{\hat{M}}, \\
& \frac{d \hat{F}_{A}}{d \hat{z}}=-\frac{\hat{F}_{A} \hat{F}_{B}}{\hat{M}}, \\
& \frac{d \hat{F}_{B}}{d \hat{z}}=\hat{M}^{1 / 2}-\gamma \frac{\hat{F}_{A} \hat{F}_{B}}{\hat{M}} .
\end{aligned}
$$

where,

$\gamma=\frac{k^{5 / 4}\left|v_{A}\right|^{1 / 4}\left|v_{B}\right| C_{B, \infty}^{1 / 4} F_{A, 0}}{2^{3 / 4} \pi^{1 / 4} \alpha^{1 / 2} B_{0}^{3 / 4}}=\frac{\left|v_{B}\right|}{\left|v_{A}\right|} \frac{F_{A, 0}}{F_{B, S}}=\varepsilon \lambda$

The boundary conditions for these five equations are 
$\hat{Q}_{0}=\frac{Q_{0}}{Q_{S}}, \hat{M}_{0}=\frac{M_{0}}{M_{S}}, \hat{B}_{0}=1, \hat{F}_{A, 0}=1, \hat{F}_{B, 0}=0$,

where the subscript 0 indicates values at the source. There are therefore four groups which influence the behaviour of the reactive plume:

$\hat{Q}_{0}, \hat{M}_{0}, \varepsilon=F_{A, 0} g_{r \times n}^{\prime} /\left(\left|v_{A}\right| B_{0}\right)$ and $\gamma$.

It is interesting to note that some of the boundary conditions above can be combined to yield familiar dimensionless groups describing plume behaviour. For example, combining equations (2.39 a) and (b) yields:

$\frac{5}{4} \frac{\hat{Q}_{0}^{2}}{\hat{M}_{0}^{5 / 2}}=\frac{5}{2^{7 / 2} \alpha \pi^{1 / 2}} \frac{B_{0} Q_{0}^{2}}{M_{0}^{5 / 2}}=\Gamma_{0}$,

This group is the inlet parameter $\Gamma_{0}$, first defined by Morton (1959) and more recently noted by Hunt and Kaye (2001), with $\Gamma_{0}=1$ corresponding to a pure plume, $\Gamma_{0}<1$ to a forced plume, which has an excess of momentum flux and $\Gamma_{0}>1$ to a distributed or lazy plume, which has a deficit of momentum flux.

Equations (2.35) and (2.36) can be combined and integrated to yield an expression relating the flux of buoyancy to the flux of species A. The buoyancy flux is given by $\hat{B}=1+\frac{F_{A, 0} g_{r x n}^{\prime}}{\left|v_{A}\right| B_{0}}\left(1-\hat{F}_{A}\right)$.

When all of species A has been exhausted, the flux of A is zero and the buoyancy flux tends to a constant value. Equation (2.42) indicates that the maximum buoyancy flux generated by the reaction is given by

$\hat{B}_{r x n}=\frac{F_{A, 0} g_{r x n}^{\prime}}{\left|v_{A}\right| B_{0}}$

As one would intuitively expect, the additional buoyancy flux generated by the reaction is given by the product of the flux of species A at the source, which governs the overall rate of the reaction in the plume, and $g_{r x n}^{\prime}$, which is a measure of the potential of the reaction to generate buoyancy. Equation (2.43) also provides a criterion for the collapse of a plume in which chemical reaction reduces the buoyancy flux. The plume will become negatively buoyant, and hence collapse, if $\hat{B}_{r x n}<-1$.

In addition to finding the buoyancy generated by the reaction, approximate analytical solutions for a reactive plume, rising from a point source of buoyancy with $\hat{Q}_{0}=\hat{M}_{0}=0$, can be found. A perturbation analysis was performed, with the small parameter $\varepsilon$ defined as 
$\varepsilon=F_{A, 0} g_{r x n}^{\prime} /\left(\left|v_{A}\right| B_{0}\right)$

Thus, solutions perturbed around a reactive plume, which does not generate buoyancy were sought. In addition, the coefficient in the reaction term of equation (2.37) was rewritten as $\gamma$ $=\varepsilon \lambda\left(\right.$ as in equation (2.38)) where $\lambda=\left|v_{B}\right| B_{0} /\left(F_{B, S} g_{r \times n}^{\prime}\right)$. If $\lambda \leq O(1)$, then $\left|v_{B}\right| F_{A, 0} /\left(\left|v_{A}\right| F_{B, S}\right) \sim O(\varepsilon)$ implying that species B is in great excess in the plume; hence, species B is only weakly consumed by the reaction and the variation of the flux of B with height is principally governed by the entrainment of ambient B into the plume.

When attempting to solve for the $O(\varepsilon)$ terms in the perturbation series for the volume and momentum fluxes, as well as the fluxes of species A and B, it was found that the governing ODEs had a singular point at the source so the appropriate boundary conditions could not be applied. This indicates the existence of a boundary layer just above the source, which has not been considered here. The integration constants in the solutions for the $O(\varepsilon)$ terms in the volume and momentum fluxes were assigned to zero to ensure the solution behaved as expected as $\hat{z} \rightarrow 0$ and $\infty$. When $\hat{z} \rightarrow 0$, both the volume and momentum fluxes should also approach zero. When $\hat{z} \rightarrow \infty$ the reaction ceases when species $\mathrm{A}$ in the plume is depleted. The buoyancy flux reaches a constant value and the volume and momentum fluxes should behave as those for an inert plume i.e. $\hat{Q} \propto \hat{z}^{5 / 3}$ and $\hat{M} \propto \hat{z}^{4 / 3}$. The $O(\varepsilon)$ terms in the perturbation series for the fluxes of species A and B were not solved for because of the singular point at the source and because the solutions would contain a very large number of terms, rendering them of little practical use.

The variation of the fluxes with height are then approximately given by

$$
\hat{Q}=\frac{3^{\frac{5}{3}} \hat{z}^{\frac{5}{3}}}{2^{\frac{2}{3}} 5^{\frac{4}{3}}}+\varepsilon\left(\frac{3^{\frac{2}{3}} \hat{z}^{\frac{5}{3}}}{2^{\frac{2}{3}} 5^{\frac{4}{3}}}+\frac{5^{\frac{4}{3}} \sqrt{\pi} \operatorname{erf}[\sqrt{\phi}]}{72^{\frac{1}{3}} 3^{\frac{2}{3}} \hat{z}^{\frac{5}{3}}}-\frac{5 \exp [-\phi]}{7 \hat{z}}-\frac{3^{\frac{1}{3}} 5^{\frac{1}{3}} \exp [-\phi] \hat{z}^{\frac{1}{3}}}{72^{\frac{1}{3}}}+\frac{3^{\frac{2}{3}} 5^{\frac{1}{6}} \hat{z}^{\frac{2}{3}} \Gamma\left[\frac{3}{4}, \phi\right]}{2^{\frac{2}{3}} 7}\right)+O\left(\varepsilon^{2}\right)
$$

$\hat{M}=\phi+\varepsilon\left(\frac{5 \exp [-\phi]}{7}+\frac{2^{\frac{1}{3}} 5^{\frac{5}{3}} \exp [-\phi]}{73^{\frac{1}{3}} \hat{z}^{\frac{4}{3}}}+\frac{2 \phi}{3}-\frac{25 \sqrt{\pi} \operatorname{erf}[\sqrt{\phi}]}{21 \hat{z}^{2}}+\frac{2^{\frac{2}{3}} 3^{\frac{1}{3}} \hat{z}^{\frac{1}{3}} \Gamma\left[\frac{3}{4}, \phi\right]}{75^{\frac{1}{6}}}\right)+O\left(\varepsilon^{2}\right)$, 


$$
\begin{aligned}
& \hat{B}=1+\varepsilon(1-\exp [-\phi])+O\left(\varepsilon^{2}\right) \\
& \hat{F}_{A}=\exp [-\phi]+O(\varepsilon) \\
& \hat{F}_{B}=\frac{3^{\frac{5}{3}} \hat{z}^{\frac{5}{3}}}{2^{\frac{2}{3}} 5^{\frac{4}{3}}}+O(\varepsilon)
\end{aligned}
$$

where $\phi=3^{\frac{4}{3}} \hat{z}^{\frac{4}{3}} / 2^{\frac{4}{3}} 5^{\frac{2}{3}}$ and $\Gamma[a, b]$ is the incomplete Gamma function.

Unlike the approximate solutions in Conroy and Llewellyn Smith (2008), the solutions in equations $(2.45)-(2.49)$ describe a plume undergoing a chemical reaction which generates buoyancy in the regions above the source where reaction is occurring, and also captures the behaviour in the far-field where species $\mathrm{A}$ has been depleted and the buoyancy flux reaches a new constant value. Equation (2.47) for the variation of buoyancy flux with height is consistent with that derived above in equation (2.42) and indicates that the buoyancy flux increases from 1 at the source to the fixed value $1+\hat{B}_{r x n}$, according to $\left(1-\exp \left(-\hat{z}^{4 / 3}\right)\right)$. This differs from the simple form assumed by Ishimine (2007), where the variation of buoyancy flux with height was proportional to $(1-\exp (-\hat{z}))$. It is also interesting to note that the increase in the volume and momentum fluxes in the far-field of the plume with buoyancy generation compared to one without can be estimated from equations (2.45) and (2.46). Equation (2.45) indicates that the volume flux in the far-field of a reactive plume differs from the inert plume by a factor of $(1+\varepsilon / 3)$, whilst equation (2.46) shows that the momentum flux differs from an inert plume by a factor of $(1+2 \varepsilon / 3)$.

Equations $(2.45)-(2.49)$ indicate that the behaviour of a reactive pure plume which generates buoyancy is principally governed by $\varepsilon=F_{A, 0} g_{r x n}^{\prime} /\left(\left|v_{A}\right| B_{0}\right)$. Conversely, the parameter $\lambda$ only appears in the higher order terms in equations $(2.45)-(2.49)$ and hence the plume behaviour will be only weakly dependent on this value. These observations and the results in equations $(2.45)-(2.49)$ are verified below by comparison with numerical solutions of the full equations $(2.33)-(2.37)$.

\section{Numerical Simulations}

The governing equations (2.33) - (2.37) were solved numerically using Mathematica. In addition to plotting the integral values from the numerical solutions, the local value of $\Gamma$, plume width and centreline velocity are also presented. The local value of $\Gamma$ is defined here as: 
$\Gamma=\frac{5}{2^{7 / 2} \alpha \pi^{1 / 2}} \frac{B Q^{2}}{M^{5 / 2}}=\frac{5}{4} \frac{\hat{B} \hat{Q}^{2}}{\hat{M}^{5 / 2}}$.

The value of this parameter will, of course, indicate regions in which the plume is behaving as a pure plume $(\Gamma=1)$, a forced plume $(\Gamma<1)$ or a lazy plume $(\Gamma>1)$. Additionally, the variation of $\Gamma$ with height may be important as there is some evidence to suggest that it may affect the magnitude of the entrainment coefficient $\alpha$. Plumes typically have greater entrainment than jets, and empirical correlations relating the local entrainment coefficient to $\Gamma$ (or some similar parameter such as $R i$ ) are well established (e.g. Fisher et al., 1979).

Recently, Kaminski et al. (2005) revisited the theoretical work of Priestley and Ball (1955) and developed a new expression relating $\alpha$ and $\Gamma$. The variation of entrainment coefficient with height is not the focus of this work and hence, for simplicity the entrainment coefficient, $\alpha$, has been assumed to be constant and equal to 0.1 . The variation of $\Gamma$ simply serves to characterise whether the plume is lazy, forced or neither at different heights in the plume as reaction proceeds.

The plume width can be calculated from the volume and momentum fluxes:

$b=Q /(2 \pi M)^{1 / 2}$,

or in dimensionless form:

$\hat{b}=b /(2 \alpha L)=\hat{Q} / \hat{M}^{1 / 2}$.

Similarly, the vertical velocity at the centreline is

$w_{g}=2 M / Q$,

or in dimensionless form

$\hat{w}_{g}=w_{g} /\left(2 k C_{B, \infty} L\right)=\hat{M} / \hat{Q}$.

Thus for a plume with no internal generation of buoyancy,

$\hat{b}=\frac{3}{5} \hat{z}$ and

$\hat{w}_{g}=\frac{5^{2 / 3}}{2^{2 / 3} 3^{1 / 3}} \hat{z}^{-1 / 3}$.

Figure 1 compares the results of numerical simulations with the approximate analytical solutions in equations $(2.45)-(2.49)$, for a pure plume issuing from a point source. Here, $\varepsilon=F_{A, 0} g_{r x n}^{\prime} /\left|v_{A}\right| B_{0}=0.25$ and $1, \lambda=\left|v_{B}\right| B_{0} /\left(F_{B, S} g_{r x n}^{\prime}\right)=1$ and $\hat{Q}_{0}=10^{-6}$ with $\Gamma_{0}=1$. The numerical scheme used to solve equations $(2.33)-(2.37)$ requires that $\hat{Q}_{0}$ is non-zero. The small value chosen ensures that the numerical results are a good approximation to a plume 
issuing from a point source of buoyancy. For both values of $\varepsilon$ the thick lines represent the numerical results and the thin lines the represent equations $(2.45)-(2.49)$.

The numerical solutions for the volume flux and momentum flux in figures 1 (a) and (b) show that equations (2.45) and (2.46) overestimate slightly the volume and momentum fluxes; nevertheless, the analytical solutions do provide a good representation of the fluxes, particularly moving into the far-field for $\hat{z}>10$. As expected, the discrepancy between the numerical and analytical solutions increases with $\varepsilon$. As mentioned above, the approximate analytical solutions for the volume and momentum fluxes are singular at the source. In fact, for $\hat{z}<10^{-5}$ the analytical solutions show $\hat{Q} \rightarrow \infty$ and $\hat{M} \rightarrow-\infty$. Whilst this behaviour is unphysical, for the regions of interest farther from the source, the agreement between the numerical and analytical solutions indicates that the solutions are applicable to all $\hat{z}$ of practical interest. Figure 1(c) shows that equation (2.47) for the buoyancy flux agrees very well with the numerical solution, both in terms of the final steady-state buoyancy flux and the length scale over which this buoyancy is generated in the plume.

When $\varepsilon=0.25$, there is good agreement between the numerical and analytical solutions for the fluxes of species A (figure 1(d)), which decays from the source value to 0, and species B (figure 1(e)), which increases with height above the source due to entrainment. When $\varepsilon$ is increased, the agreement between the analytical and numerical solutions, particularly for the flux of $\mathrm{A}$, is less good than at lower $\varepsilon$. In fact, the analytical solutions for each $\varepsilon$ are identical because the $O(\varepsilon)$ terms for the fluxes of $\mathrm{A}$ and $\mathrm{B}$ have not been found. The poorer agreement between the numerical and analytical results is therefore expected.

There is a discrepancy between the analytical and numerical solutions for $\Gamma$ for small $\hat{z}$. This is due to the existence of the unaccounted for boundary layer in the solutions for volume and momentum flux, as discussed above. For $\hat{z}>0.5$, figure 1(f) shows reasonable agreement between the numerical and analytical results. It is evident that $\Gamma$ increases above unity due to the buoyancy generated by the reaction i.e. the plume becomes lazy. When species $\mathrm{A}$ is exhausted and the buoyancy flux has reached its new constant value, $\Gamma$ decays back to one. Unlike the approximate solutions in Conroy and Llewellyn Smith (2008), this behaviour is captured by the approximate analytical solutions in equations $(2.45)-(2.49)$.

Figure $1(\mathrm{~g})$ shows that the analytical solutions overestimate slightly the width of the plume. It is also worth noting that whilst the numerical results show a slight narrowing of the plume with increasing $\varepsilon$, the analytical approximations indicate the opposite effect. Figure 1(h) shows that whilst there is some difference between the centreline velocity predicted by 
equations $(2.45)-(2.49)$ in the region close to the source, in the far-field there is excellent agreement between the analytical and numerical solutions for the plume velocity.

Equations $(2.45)-(2.49)$ can also be used when $\varepsilon<0$. The analytical solutions agree very well with the numerical simulations, particularly in the far-field. There is as discussed above some discrepancy near the source. As $\varepsilon \rightarrow-1$ the agreement between the numerical and analytical solutions becomes much less good due to the increasing value of $|\varepsilon|$.

\subsection{Effects of $\varepsilon=F_{A, 0} g_{r x n}^{\prime} /\left|v_{A}\right| B_{0}$}

Equations (2.43) and (2.47) indicate that increasing $\varepsilon=F_{A, 0} g_{r x n}^{\prime} /\left|v_{A}\right| B_{0}$ increases the buoyancy generated by the reaction. The effects of increasing $\varepsilon$ are shown in figure 2 for cases with:

$\gamma=\frac{k^{5 / 4}\left|v_{A}\right|^{1 / 4}\left|v_{B}\right| C_{B, \infty}^{1 / 4} F_{A, 0}}{2^{3 / 4} \pi^{1 / 4} \alpha^{1 / 2} B_{0}^{3 / 4}}=1, \hat{Q}_{0}=10^{-6}$ and $\Gamma_{0}=1$.

In each case presented in figure 2 , the total buoyancy generated by the reaction is consistent with equation (2.43). Figure 2(a) and (b) show log-log plots of the volume and momentum fluxes. When $\varepsilon=0.1$, there is clearly very little deviation from the expected power-law variations for an inert plume, i.e. $\hat{Q} \propto \hat{z}^{5 / 3}$ and $\hat{M} \propto \hat{z}^{4 / 3}$. This is unsurprising given that the reaction generates a small amount of buoyancy relative to that released at the source. It is evident that the volume and momentum fluxes for the cases with $\varepsilon=1$ and 10 are significantly larger than those seen for $\varepsilon=0.1$. Figure 2(a) and (b) also show the volume and momentum fluxes in the plume returning to the familiar power-law forms in the far field once the reaction has depleted the plume of species A, as predicted in equations (2.45) and (2.46). In fact, figure 2(a) and (b) show the plume returning to the power law behaviour with $\hat{Q} \propto \hat{z}^{5 / 3}$ and $\hat{M} \propto \hat{z}^{4 / 3}$ for $\hat{z}>\sim 20$.

The fluxes of A and B in figure 2(d) and (e) reveal some interesting trends. As expected, the flux of A tends to zero over a finite height above the source. When the flux of A becomes zero, the buoyancy flux reaches a constant value. The length scale over which the flux of A tends to zero can be seen from figure 2(d) to depend only very weakly on $\varepsilon$, with larger values of $\varepsilon$ resulting in plumes in which the flux of A tends to zero over a slightly larger height above the source. Figure 2(e) shows that the flux of B increases as $\varepsilon$ increases. This is because the increased buoyancy in the plume leads to an increased velocity. This 
increased vertical velocity leads to more ambient fluid, and hence more species B, being entrained into the plume according to equation (1.1).

Unsurprisingly, for each case considered in figure 2(f), the maximum $\Gamma$ is attained in the plume which generates the maximum buoyancy. It is also evident that the height at which the maximum $\Gamma$ is reached has only a slight dependence on $\varepsilon$. The magnitudes of the increases in $\Gamma$ are also noteworthy. If $\Gamma>5 / 4$ the plume velocity will actually increase with height and if $\Gamma>5$ / 2 the plume width will decrease with height (Caulfield, 1991). Even with $\varepsilon=10$, i.e. a plume in which the reaction generates ten times the buoyancy flux released at the source, the maximum value of $\Gamma$ increases by less than $25 \%$ and so the effect of the reaction is insufficient to cause the plume velocity to increase with height, or to cause the plume to contract with height.

Figure $2(\mathrm{~g})$ and $(\mathrm{h})$ show the difference between the plume width and centreline velocity in a plume which generates buoyancy compared with one which does not (equations (3.6) and (3.7)) versus height, for different values of $\varepsilon$. Figure $2(\mathrm{~g})$ shows that when the reaction generates buoyancy, the plume is narrower than an inert plume. As the reaction ceases and the buoyancy flux reaches a constant value, the difference in width between a reactive and inert plume tends to a constant value; hence the gradient of $\hat{b}$ versus $\hat{z}$ tends to the expected far-field value of $3 / 5$. This narrowing of the plume with additional buoyancy generated by the reaction agrees well with previous experimental and theoretical investigations (e.g. Hunt and Kaye, 2005), as well as with the similarity solutions derived by Batchelor (1954) for an inert plume rising in an unstably stratified environment, discussed above. The behaviour is slightly different from that discussed by Scase et al. (2006) for a plume with a time dependent buoyancy flux, where the plume width far from the source is necessarily given by the similarity solution of Morton et al. (1956). However, figure 2(g) does show that the plume with chemical reaction is only very slightly narrower than an inert plume. As expected, the dimensionless vertical velocity at the centreline (figure $2(\mathrm{~h})$ ) is larger with an increase in $\varepsilon$. As is the case for the maximum value of $\Gamma$, the maximum difference between the velocities of a reactive and inert plume is only very weakly dependent on the value of $\varepsilon$. It should also be noted that, as discussed above, the plume fluid decelerates as it moves away from the source in each case because $\Gamma$ never exceeds $5 / 4$.

In summary, figure 2 shows that the parameter $\varepsilon$ affects the buoyancy generated by the reaction but has very little effect on the height above the source over which reaction occurs. The increase in buoyancy generation for larger values of $\varepsilon$ results in larger peak values of $\Gamma$, 
but even when the reaction generates significant additional buoyancy, the reaction is insufficient to cause the plume to become narrower with distance from the source or to cause the plume velocity to increase with height.

3.2 Effects of $\gamma=\frac{k^{5 / 4}\left|v_{A}\right|^{1 / 4}\left|\nu_{B}\right| C_{B, \infty}^{1 / 4} F_{A, 0}}{2^{3 / 4} \pi^{1 / 4} \alpha^{1 / 2} B_{0}^{3 / 4}}$

Figure 3 shows numerical results for increasing values of $\gamma$ when $\varepsilon=1$ and $\hat{Q}_{0}=10^{-6}$ with $\Gamma_{0}=1$. The group $\gamma$ only appears in the reaction term in equation (2.37) for the flux of B. As discussed above, when $\gamma$ is small, the coefficient of the reaction term is small and entrainment dominates the variation of the flux of B with distance from the source. Figure 3(a) and (b) show that for the cases with $\gamma=0.1$ and 1, there is very little difference between the volume and momentum fluxes of the two plumes. Figure 3(c) and (d) show that there is only a very slight increase in the height over which reaction occurs when $\gamma$ is increased from 0.1 to 1 . This increase in the height over which reaction is important is also evident in Figure 3(f) for $\Gamma$.

When $\gamma$ is increased to 10 however, the reaction term becomes important and species B is very strongly depleted by the reaction. This is most evident in figure 3(e), where the flux of $\mathrm{B}$ is considerably lower when $\gamma=10$ than in the other cases considered. In fact, there is a region near the source $(\hat{z}<\sim 3)$ over which the flux of $\mathrm{B}$ is very small. In this region virtually all of species B which is entrained into the plume is consumed by reaction. This means that there is a region above the source where the reaction is controlled by entrainment as opposed to the chemical kinetics. The reduced flux of B when $\gamma=10$ results in a lower rate of reaction; thus, the flux of A drops to zero over a larger distance than is the case for lower values of $\gamma$, and similarly, the buoyancy flux reaches its constant value farther from the source. The reduced rate of reaction also results in the volume and momentum fluxes being marginally lower than was the case for the smaller values of $\gamma$.

The maximum value of $\Gamma$ is reached at a larger $\hat{z}$ for larger values of $\gamma$ as evident in figure 3(f). It is also interesting to note that the maximum value of $\Gamma$ does increase slightly when $\gamma$ is increased. Figure $3(\mathrm{~g})$ indicates that the reactive plume is narrower than the inert equivalent for all values of $\gamma$ considered. The longer length above the source over which reaction occurs results in the plume being narrower for larger values of $\gamma$. Figure 3(h) shows that the differences between the centreline velocity of a reactive plume and inert plume for 
the three values of $\gamma$ are very similar. The differing rates of buoyancy release result in lower velocities near the source for larger values of $\gamma$. In the far-field, the velocity in all three cases is virtually identical. The reduced volume and momentum fluxes for larger $\gamma$ are therefore due to the fact that the plume is narrower when $\gamma$ is increased.

Comparison of the results of sections 3.1 and 3.2 indicate that whilst the additional buoyancy generated by the reaction is governed by $\varepsilon$, the height over which the reaction occurs is principally controlled by $\gamma$, providing $\gamma$ is sufficiently large.

\subsection{Effects of $\varepsilon$ and $\gamma$ on the maximum $\Gamma$ attained in the plume}

It was noted above that the values of the parameters $\varepsilon$ and $\gamma$ affected the maximum value of $\Gamma$ attained in the plume as well as the height above the source at which this maximum was reached. Plotted in figure 4 are (a) the distance from the source at which $\Gamma_{\max }$ occurs and (b) $\Gamma_{\max }$ against $\varepsilon$ for three different values of $\gamma$. The maximum value of $\varepsilon$ in figure 4 is 10 , which from equation (2.43) corresponds to a plume where the additional buoyancy flux generated by chemical reaction is ten times the buoyancy flux at the source. It is of course questionable if the Boussinesq approximation holds for such large values of $\varepsilon$. The values of $\gamma$ chosen are 0 , which corresponds to the notional limit where species B is not depleted by reaction, 1 and 10, where species B is strongly depleted by reaction. Figure 4(a) shows that for each value of $\gamma$, the variation of $\hat{z}$ at $\Gamma_{\max }$ decreases very slightly with increased $\varepsilon$. The height at which this maximum occurs is also weakly dependent on $\gamma$ for reactions in which species B is only weakly depleted $(0<\gamma<1)$ but does increase significantly with $\gamma$ for $\gamma>1$ when the depletion of species B by the reaction becomes significant. As expected, figure 4(b) shows that $\Gamma_{\max }$ increases with $\varepsilon$ for each $\gamma$ because of the increased buoyancy generated by the reaction. As noted in section 3.2, $\Gamma_{\max }$ is larger for larger values of $\gamma$. For a fixed $\varepsilon$, the buoyancy flux generated by the reaction is the same; however, figure 3(a) and (b) show that a larger value of $\gamma$ results in smaller volume and momentum fluxes than is the case for small $\gamma$. Equation (3.1) indicates that it must be the reduction in momentum flux which causes the higher values of $\Gamma_{\max }$.

\subsection{Virtual source calculation for the far-field plume}

It was noted above that when all of species A has been consumed by the reaction, the volume and momentum fluxes tend toward the power-law variations expected for a pure, 
inert plume. Given that the plume has a constant buoyancy flux in the far-field, $\hat{B}_{\infty}=1+\varepsilon$, an equivalent pure, inert plume issuing from a virtual source located away from the real source can be found which matches the behaviour of the reactive plume far away from the source. The fluxes of volume, momentum and buoyancy can therefore be considered to be of the form:

$$
\hat{Q}=\frac{3^{\frac{5}{3}}}{2^{\frac{2}{3}} 5^{\frac{4}{3}}} \hat{B}_{\infty}^{\frac{1}{3}}\left(\hat{z}-\hat{z}_{v}\right)^{\frac{5}{3}} ; \hat{M}=\frac{3^{\frac{4}{3}}}{2^{\frac{4}{3}} 5^{\frac{2}{3}}} \hat{B}_{\infty}^{\frac{2}{3}}\left(\hat{z}-\hat{z}_{v}\right)^{\frac{4}{3}} ; \hat{B}=\hat{B}_{\infty}=1+\varepsilon,
$$

where $\hat{z}_{v}$ is the location of the virtual source.

It should be recalled from section 2.3 that the approximate analytical solutions indicated that for large $\hat{z}$, the volume flux was larger than that for an inert plume by a factor of $(1+\varepsilon / 3)$, whereas the momentum flux was larger by a factor of $(1+2 \varepsilon / 3)$. It is clear that these factors are the first two terms in the series expansion of $\hat{B}_{\infty}^{1 / 3}$ and $\hat{B}_{\infty}^{2 / 3}$ from equations (3.8) and (3.9). Comparison of equations (3.8) and (3.9) with the forms of the approximate analytical solutions in equations (2.45) and (2.46) also indicate that $\hat{z} / \hat{z}_{v}>1$, i.e. the virtual source is located very close to the real source compared with the distance over which power-law behaviour in the far field is re-established.

In order to confirm the forms of equations (3.8) and (3.9), the location of the virtual source was found numerically when $\varepsilon$ and $\gamma$ were varied. The variation of the location of the virtual source with $\varepsilon$ for $\gamma=0,1,5$ and 10 is shown in figure 5. In each case, it is clear that $\hat{z}_{v}>0$, i.e. the virtual source is always located ahead of the real source. The fact that the virtual source is downstream of the real source is as expected, given the increases in volume and momentum fluxes associated with the chemical reaction, and the associated reduction in the plume width. Figure $2(\mathrm{~g})$ shows that plumes with large $\varepsilon$ are narrower than those with small $\varepsilon$, hence, one would expect the virtual source to move farther from the real source with increasing $\varepsilon$ and this also evident in figure 5. A similar trend emerges for the effect of varying $\gamma$. Figure $3(\mathrm{~g})$ shows that larger values of $\gamma$ result in narrower plumes, and hence virtual sources which are farther from the real source as shown clearly in figure 5.

It is also interesting to note that for the for the cases presented in figure $5, \hat{z}_{v} \sim O(1)$. This means that $z_{v}$ is order $L$, i.e. the virtual source is located ahead of the real source by a similar distance to the characteristic length scale over which reaction is important. Figures 2(f) and 3(f) show that the maximum value of $\Gamma$ is also reached over a similar length scale. 
Figures 2(f) and 3(f) also show that for plumes with reactions which generate significant additional buoyancy, $\Gamma \rightarrow 1$ for $\hat{z}>\sim 10$, thus confirming the above hypothesis that the virtual source is located close to the real source, relative to the distance over which powerlaw behaviour is re-established in the far-field.

\subsection{Effects of the volume flux at the source $\hat{Q}_{0}$}

Thus far, only a plume issuing from a point source of buoyancy has been considered. Many real plumes have non-zero fluxes of volume and momentum at the source i.e. $\hat{Q}_{0}$ and $\hat{M}_{0}$ are non-zero. The behaviour of such plumes is considered below. It should be noted that the length scale derived in equation (2.28) has been used here for consistency. Of course, for plumes with non-zero volume and momentum fluxes at the source, the familiar length scales $L_{Q}\left(\sim Q_{0}^{3 / 5} / B_{0}^{1 / 5}\right)$ and $L_{M}\left(\sim M_{0}^{3 / 4} / B_{0}^{1 / 2}\right)$, which indicate the height above the source over which the volume and momentum fluxes at the source are important, can be derived. The effect of increasing the volume flux $\hat{Q}_{0}$ and momentum flux $\hat{M}_{0}$ simultaneously (with $\Gamma_{0}=$ 1) on the behaviour of the plume is shown in figure 6 . Here $\hat{Q}_{0}$ is varied from $0.1-10$ with $\varepsilon$ $=\gamma=1$.

The numerical results in figure 6(a) and (b) show that increasing $\hat{Q}_{0}$ has little effect on the development of the volume and momentum fluxes with height just above the source, beyond the increase in their magnitudes. However, for larger values of $\hat{Q}_{0}$ the plume approaches the familiar power-law variations of volume and momentum flux farther from the source. Figure 6(c) and (d) indicate that the length scale over which reaction occurs is largely unaffected by the increase in $\hat{Q}_{0}$; the decrease in the flux of A with height and the consequent increase in buoyancy flux are very similar in all three cases presented.

Despite the fact that each case considered in figure 6 generates the same additional buoyancy flux over approximately the same height above the source, larger values of $\hat{Q}_{0}$ (and hence, $\hat{Q}$ ) result in a larger maximum value of $\Gamma$ in figure $6(\mathrm{f})$. It should be recalled from section 3.1 that when $\varepsilon=10$ in a plume rising from a point source of buoyancy the maximum value of $\Gamma$ was less than $5 / 4$. In this case with $\varepsilon=1$, a larger value of $\hat{Q}_{0}=10$ results in $\Gamma_{\max }$ $\sim 1.35$. 
The plots for the variation of the dimensionless plume width with height in figure $6(\mathrm{~g})$ show a similar trend to those seen previously in figure 2, i.e. in the region above the source for plumes with strong generation of buoyancy, the plume is slightly narrower than an inert plume would be. Despite the larger values of $\Gamma_{\max }$ than were seen in figure 2, the plume is still far removed from the conditions which would cause the plume width to decrease with height $(\Gamma>5 / 2)$. In the far-field, the variation of plume width with height is linear with a gradient consistent with the result for pure, inert plumes. It should be pointed out that the different intercepts at $\hat{z}=0$ occur due to the source having finite size for $\Gamma_{0}=1$ and $\hat{Q}_{0} \neq 0$.

For a similar reason, the dimensionless velocity is lower for larger values of $\hat{Q}_{0}$ in figure $6(\mathrm{~h})$. When the volume flux at the source is small $\left(\hat{Q}_{0}=0.1\right)$, the velocity decays very rapidly above the source. It is interesting to note that when $\hat{Q}_{0}=10$, the velocity is approximately constant as the distance from the source increases. Closer inspection reveals that there is in fact a region in which the velocity is increasing with distance from the source. This increase in velocity with height corresponds to the region in which $\Gamma>5 / 4$ in figure 6(f) (Caulfield, 1991). In this case, the reaction generates sufficient additional buoyancy to accelerate the plume, which is qualitatively similar to the behaviour predicted by Batchelor's (1954) solutions for an inert plume rising in an unstably stratified environment.

In summary, varying $\hat{Q}_{0}$ can be seen from figure 6 to have little effect on the height over which chemical reaction occurs in the plume. The main effect of increasing $\hat{Q}_{0}$ is the increase in the maximum value of $\Gamma$ due to the larger value of the factor $5 \hat{Q}^{2} /\left(4 \hat{M}^{5 / 2}\right)$ in equation (3.1). It is evident from comparison of figure 2(f) and figure 6(f) that the maximum value of $\Gamma$ is increased more by increasing the volume flux at the source than by increasing $\varepsilon$, i.e. the buoyancy generated by the reaction.

\subsection{Effects of $\Gamma_{0}$}

In many important cases, the plume will not have $\Gamma_{0}=1$ at the source, as considered above. If $\Gamma_{0}<1$ the plume has an excess of momentum flux at the source and is behaving as a forced plume, whereas if $\Gamma_{0}>1$, the plume has a deficit of momentum flux at the source and is a lazy plume. The effects of varying $\Gamma_{0}$ are considered below. In practice, varying $\Gamma_{0}$ could be achieved by passing the same volume flux $\hat{Q}_{0}$ through a source of varying size. 
Figure 7 shows the behaviour of several forced plumes with buoyancy generation by chemical reaction. Here $\hat{Q}_{0}=\varepsilon=\gamma=1$. The log-log plots of volume and momentum flux in figure 7(a) and (b) show that the cases with a lower $\Gamma_{0}$, i.e. those with a higher degree of forcing, have larger volume and momentum fluxes above the source. It is also evident in Figure 7(a) and (b) that the magnitude of the volume and momentum fluxes in the far field is very similar for each case considered, and that the height at which the plume approaches the far-field behaviour, with $\hat{Q} \propto \hat{z}^{5 / 3}$ and $\hat{M} \propto \hat{z}^{4 / 3}$, is increased when the degree of forcing in the plume is increased.

As expected, varying $\Gamma_{0}$ has no effect on the buoyancy flux generated by the reaction and figure 7(c) and (d) show that there is very little difference in the height over which buoyancy is generated in the plume between each of the cases presented. Figure 7(f) shows the variation of $\Gamma$ with height above the source. When $\Gamma_{0} \sim 1$, the buoyancy generated by the reaction causes $\Gamma$ to increase above 1, i.e. the reaction still causes the plume to become lazy over a limited height, before $\Gamma$ tends to unity in the far field. For $\Gamma_{0}$ closer to zero, $\Gamma$ approaches the pure plume limit monotonically. Figures $7(\mathrm{~g})$ and $(\mathrm{h})$ show that cases with a smaller $\Gamma_{0}$ have a smaller plume width at the source and higher velocity. For the cases with $\Gamma_{0}<1$ in figure $7(\mathrm{~g})$, the rate of spread of the plume just above the source is larger than that seen in a pure plume, as expected.

Figure 8 compares the behaviour of several lazy plumes (i.e. $\Gamma_{0}>1$ ). It is very clear that the behaviours of the plumes in all the cases considered are very similar. There are no significant differences between the variation of the volume, momentum and buoyancy fluxes for any of the cases presented. Figure 8(f) shows that in a lazy plume, $\Gamma$ decays to unity as height increases. Figure $8(\mathrm{~g})$ and $(\mathrm{h})$ show some features which characterise lazy plumes. Just above the source the plume radius actually decreases slightly for $\Gamma_{0}=5$ and 10 . As discussed above, this narrowing occurs if $\Gamma>5$ / 2 (Caulfield, 1991). Similarly, for the three cases with $\Gamma_{0}>5 / 4$ the plume fluid accelerates as it moves away from the source, reaching a maximum when $\Gamma=5 / 4$, before decaying to the far-field solution, which is virtually identical for all of the cases considered.

Consideration of both figure 7 and 8 reveals that varying $\Gamma_{0}$ has very little effect on either the length scale over which reaction occurs in the plume or the behaviour of the plume in the far field. Varying $\Gamma_{0}$ does impact on the plume behaviour just above the source, as it does for inert plumes. 


\subsection{Reversing buoyancy}

Up to this point, only reactions which cause an increase in buoyancy flux have been considered (i.e. reactions in which the products are less dense than the reactants or exothermic reactions); however, reactions which reduce the buoyancy flux in the plume, such as endothermic reactions or reactions in which the products are more dense than the reactants, can also occur. In such a plume the buoyancy flux will be reduced to zero at some height above the source when $\hat{B}_{r x n}=-1$. If $\hat{B}_{r x n}$ is reduced further the plume will become negatively buoyant and may subsequently collapse.

The governing equations can be solved for a plume with reversing buoyancy. Such a plume can be achieved by having $B_{0}$ and $g_{r x n}^{\prime}$ of different sign. Figure 9 compares the behaviour of a plume from a point source of buoyancy with $\hat{B}_{r x n}=-1$ (i.e. a plume where the buoyancy flux is reduced to zero at some height above the source) with that of a plume where $\hat{B}_{r x n}=-1.1$. Figure 9(c) shows that for $\hat{B}_{r x n}=-1$ the buoyancy flux in the plume is effectively zero for $\hat{z}>5$. At greater distances from the source, the momentum flux is constant, as expected, so in fact the flow is now behaving as a jet, i.e. the buoyancy flux is zero. This is reflected in the variation of the volume flux with height (figure 9(a)), which is linear as predicted for a jet. The fact that the flow has become a jet is also shown by $\Gamma$ in figure 9(f), which of course reaches zero when the buoyancy flux (see equation (3.1)) goes to zero. The value of $\Gamma$ appears to approach zero over a longer length scale in figure $9(f)$ than does the buoyancy flux in figure $9(\mathrm{c})$. This is of course due to the additional factor $5 \hat{Q}^{2} /\left(4 \hat{M}^{5 / 2}\right)$ in equation (3.1). In the region $\hat{z}>5$ the value of the entrainment coefficient $\alpha$ for a jet would be more appropriate than that for a plume; however, this has not been considered here. The plots of plume width (figure $9(\mathrm{~g})$ ) and velocity (figure $9(\mathrm{~h})$ ) also show the expected behaviour for a jet, for $\hat{B}_{r x n}=-1$. In the far-field the plot of $\hat{b}$ against $\hat{z}$ is linear with gradient 1 indicating that $b \propto 2 \alpha z$ as predicted for a jet. Similarly, the centreline velocity $\propto \hat{z}^{-1}$ as expected for a jet. When $\hat{B}_{r x n}=-1.1$, the plume collapses. This collapse occurs at $\hat{z} \sim 6.5$, where the momentum flux in the plume becomes zero and the plume radius tends to infinity, while the velocity rapidly approaches zero. This collapse is caused by the buoyancy in the plume becoming negative for $\hat{z}>3.5$. When the buoyancy flux becomes negative so does $\Gamma$ (not plotted) and as the momentum flux approaches zero, $\Gamma \rightarrow-\infty$. The 
reversal of buoyancy results in a marginally reduced volume flux. Of course, in this region the entrainment assumption in equation (1.1) becomes more questionable.

It was mentioned above that an analogy could be made between the effects of a chemical reaction which generates buoyancy and an inert plume rising in a stratified environment. For cases with $\hat{B}_{r x n}>0$, the additional buoyancy generated by the chemical reaction is analogous to a plume rising through an unstably stratified environment (i.e. one in which the density increases with height) with the density tending to a constant value for large distances from the source. Similarly, if $\hat{B}_{r x n}<-1$ the plume will collapse as shown in figure 9. This behaviour is similar to that seen when an inert plume rises in a stably stratified environment. In such situations it has been established experimentally (Turner, 1973) that the maximum rise height of the plume is given by

$z_{\max }=5.0 \pi^{-1 / 4} B_{0}^{1 / 4} N^{-3 / 4}$.

The rise height (defined here as the height at which the momentum flux is zero) of a plume which collapses due to the effects of chemical reaction is plotted as a function of $\varepsilon$, the additional buoyancy generated by the reaction, and $\gamma$, the dimensionless rate of depletion of species $\mathrm{B}$ by the reaction, in figure 10 . As expected, as $\varepsilon \rightarrow-1$, the maximum rise height tends to infinity. It is also evident from figure 10 that providing $|\varepsilon|$ is large enough, the rise height for a fixed value of $\gamma$ is only a very weak function of $\varepsilon$. For large values of $|\varepsilon|$ the maximum rise height can be approximated as $\hat{z}_{\max }=f(\gamma)$ and so for a fixed value of $\gamma$ $z_{\max } \sim B_{0}^{1 / 4}\left(\left|v_{A}\right| k C_{B, \infty}\right)^{-3 / 4}$

which is very similar in form to equation (3.11).

\section{Conclusions}

An integral model has been developed to describe the behaviour of a plume with internal generation of buoyancy, by means of a second-order chemical reaction between species $\mathrm{A}$ in the plume and species B in the environment. The potential for a reaction to generate buoyancy in a plume is measured by the term $g_{r x n}^{\prime}$, which indicates the change in reduced gravity induced by reaction. There are two contributions to $g_{r x n}^{\prime}$, one to account for thermal effects and one to account for compositional changes. Scaling indicates that the behaviour of a reactive plume depends on the volume and momentum fluxes at the source, and the groups $\varepsilon=F_{A, 0} g_{r x n}^{\prime} /\left(\left|v_{A}\right| B_{0}\right)$ and $\gamma=k^{5 / 4}\left|v_{A}\right|^{1 / 4}\left|v_{B}\right| C_{B, \infty}^{1 / 4} F_{A, 0} /\left(2^{3 / 4} \pi^{1 / 4} \alpha^{1 / 2} B_{0}^{3 / 4}\right)$. An 
expression relating the buoyancy flux in the plume to the flux of species A was derived; this equation indicates that the maximum buoyancy flux generated by the reaction is given by $B_{r x n}=F_{A, 0} g_{r x n}^{\prime} /\left|v_{A}\right|$. Approximate analytical solutions have been found for a plume rising from a point source of buoyancy. These solutions show that $\varepsilon$ is important in determining the buoyancy generated by the reaction.

The analytical solutions were verified by comparison with numerical solutions of the full governing equations. The numerical solutions showed that even for a plume with $\varepsilon=10$ i.e. one in which the buoyancy generated by the reaction is ten times that released at the source, the buoyancy generated by the reaction was insufficient to cause the plume fluid to accelerate as it moved away from the source or to cause the plume to become narrower with height. It was also shown that if $\gamma$ is small, then it has little impact on the behaviour of the plume; however, when $\gamma>1$ the height over which reaction occurs increases significantly with increasing $\gamma$. The behaviour of the plume in the far field, when reaction has ceased, was shown to be consistent with that of an inert plume with buoyancy flux $1+\varepsilon$ issuing from a virtual source downstream of the real source. Increasing $\varepsilon$ and $\gamma$ caused the location of the virtual source to move farther away from the real source.

The effects of varying the volume flux at the source and the Morton source parameter $\Gamma_{0}$, were investigated numerically. It was observed that variation of the conditions at the source had very little impact on the length scale over which reaction occurred in the plume. Interestingly, for a case with $\varepsilon=1$ it was shown that if the dimensionless volume flux at the source is increased to 10 , the effect of the reaction is sufficient to cause the velocity of the plume to increase with distance from the source. Finally, it was observed that when $\hat{B}_{r x n}$ is negative, the reaction causes a reduction in the buoyancy flux in the plume. If $\hat{B}_{r x n}=-1$, the buoyancy flux is reduced to zero above the source and the plume behaves as a jet above this height. A further small decrease in $\hat{B}_{r x n}$ is seen to result in the plume collapsing.

The financial support of the Leverhulme Trust (Grant no. F/09 844/C) is gratefully acknowledged. A.N.C. is also grateful to Girton College, Cambridge for the award of the Hertha Ayrton Research Fellowship in Chemical Engineering.

\section{References}


Agrawal, A. \& Prasad, A. K. 2004 Evolution of a turbulent jet subjected to volumetric heating. J. Fluid Mech. 511, 95 - 123.

Agrawal, A., Sreenivas, K. R. \& Prasad, A. K. 2004 Velocity and temperature measurements in an axisymmetric turbulent jet with cloud-like off-source heating. Int. J. Heat Mass Tran. $47,1433-1444$.

Batchelor, G. K. 1954 Heat convection and buoyancy effects in fluids. Q. J. Roy. Meteor. Soc. 80, $339-358$.

Bhat, G. S. \& Narasimha, R. 1996 A volumetrically heated jet: large-eddy structure and entrainment characteristics. J. Fluid Mech. 325, 303 - 330.

Bhat, G. S., Narasimha, R. \& Arakeri, V. H. 1989 A new method of producing local enhancement of buoyancy in liquid flows. Exp. Fluids 7, $99-102$.

Caulfield, C. P. 1991 Stratification and buoyancy in geophysical flows. PhD thesis, University of Cambridge.

Caulfield, C. P. \& Woods, A. W. 1995 Plumes with non-monotonic mixing behaviour. Geophys. Astro. Fluid 79, 173 - 199.

Clarke, J. F. \& McChesney, M. 1964 The Dynamics of Real Gases. Butterworths.

Conroy, D. T., Llewellyn Smith, S. G. \& Caulfield, C. P. 2005 Evolution of a chemically reacting plume in a ventilated room. J. Fluid Mech. 537, $221-253$.

Conroy, D. T. \& Llewellyn Smith, S. G. 2008 Endothermic and exothermic chemically reacting plumes. J. Fluid Mech. 612, $291-310$.

Dahm, W. J. A. 2005 Effects of heat release on turbulent shear flows. Part 2. Turbulent mixing layers and the equivalence principle. J. Fluid Mech. 540, 1-19.

Diez, F. J. \& Dahm, W. J. A 2007 Effects of heat release on turbulent shear flows. Part 3. Buoyancy effects due to heat release in jets and plumes. J. Fluid Mech. 575, $221-255$. Fischer, H. B., List, E. J., Koh, R. C. Y., Imberger, J. \& Brooks, N. H. 1979 Mixing in Inland and Coastal Waters. Academic

Hanna, S. R., Chang, J. C. \& Zhang, X. J. 1997 Modelling accidental releases to the atmosphere of a dense reactive chemical (uranium hexafluoride). Atmos. Environ. 31, 901 908.

Hunt, G. R. \& Kaye, N. G. 2001 Virtual origin correction for lazy turbulent plumes. J. Fluid Mech. 435, 377 - 396.

Hunt, G. R. \& Kaye, N. B. 2005 Lazy plumes. J. Fluid Mech. 533, 329 - 338.

Ishimine, Y. 2007 A simple integral model of buoyancy-generating plumes and its application to volcanic eruption columns. J. Geophys. Res. 112, B03210. 
Kaminski, E., Tait, S. \& Carazzo, G. 2005 Turbulent entrainment in jets with arbitrary buoyancy. J. Fluid Mech. 526, 361 - 376.

List, E. J. 1982 Turbulent jets and plumes. Ann. Rev. Fluid Mech. 14, 189 - 212.

Morton, B. R. 1959 Forced plumes. J. Fluid Mech. 5, 151 - 163.

Morton, B. R., Taylor, G. I. \& Turner, J. S. 1956 Turbulent gravitational convection from maintained and instantaneous sources. Proc. R. Soc. Lond. A 234, 1 - 23.

Priestley, C. H. B. \& Ball, F. K. 1955 Continuous convection from an isolated source of heat. Q. J. Roy. Meteor. Soc. 81, $144-157$.

Scase, M. M., Caulfield, C. P., Dalziel, S. B. \& Hunt, J. C. R. 2006 Time-dependent plumes and jets with decreasing source strengths. J. Fluid Mech. 563, $443-461$.

Tacina, K. M. \& Dahm, W. J. A. 2000 Effects of heat release on turbulent shear flows. Part 1. A general equivalence principle for nonbuoyant flows and its application to turbulent jet flames. J. Fluid Mech. 415, 23-44.

Turner, J. S. 1973 Buoyancy effects in fluids. Cambridge University Press.

Venkatakrishnan, L., Bhat, G. S. \& Narasimha, R. 1999 Experiments on a plume with offsource heating: implications for cloud fluid dynamics. J. Geophys. Res. 104, 14271 - 14281. Williams, F. A. 1985 Combustion Theory, $2^{\text {nd }}$ Edition, Benjamin/Cummings Woods, A. W. \& Caulfield, C. P. 1992 A laboratory study of explosive volcanic eruptions. J. Geophys. Res. 97, $6699-6712$.

\section{Figure Captions}

Figure 1. Comparison of the numerical (thick lines) and approximate analytical (thin lines) solutions to the governing equations. Shown are the variation with height of: (a) the dimensionless volume flux $\hat{Q}$, (b) momentum flux $\hat{M}$, (c) buoyancy flux $\hat{B}$, (d) flux of species $\mathrm{A}, \hat{F}_{A}$, (e) flux of species $\mathrm{B}, \hat{F}_{B}$, (f) the local value of $\Gamma$, (g) the plume width $\hat{b}$ and (h) the centreline velocity $\hat{w}_{g}$ for $\varepsilon=0.25$ (solid lines) and 1 (dashed lines), $\lambda=1, \hat{Q}_{0}=10^{-6}$ and $\Gamma_{0}=1$.

Figure 2. Variation of: (a) the dimensionless volume flux on log-log scales, (b) momentum flux on log-log scales, (c) buoyancy flux, (d) flux of species A, (e) flux of species B, (f) the local value of $\Gamma,(\mathrm{g})$ the difference between the width of a reactive and inert plume and $(\mathrm{h})$ 
the difference between the centreline velocity of a reactive and inert plume, for $\gamma=1, \hat{Q}_{0}=$ $10^{-6}, \Gamma_{0}=1$ and $\varepsilon=0.1,1,10$. In parts (a) and (b) the dotted lines represent the power-law variations for inert plumes.

Figure 3. Variation of: (a) the dimensionless volume flux, (b) momentum flux, (c) buoyancy flux, (d) flux of species A, (e) flux of species B, (f) the local value of $\Gamma$, (g) the difference between the width of a reactive and inert plume and (h) the difference between the centreline velocity of a reactive and inert plume, for $\varepsilon=1, \hat{Q}_{0}=10^{-6}, \Gamma_{0}=1$ and $\gamma=0.1,1,10$.

Figure 4. Variation of: (a) the height at which the maximum value of $\Gamma$ is reached and (b) the maximum value of $\Gamma$ as a function of $\varepsilon$. Here, $\hat{Q}_{0}=10^{-6}, \Gamma_{0}=1$ and $\gamma=0,1,10$.

Figure 5. Variation of the location of the virtual source $\hat{z}_{v}$ for a pure, inert plume whose behaviour is the same as a reactive plume in the far field, as a function of $\varepsilon$. The location of the virtual source was found by fitting equations (3.8) and (3.9) to numerical solutions of plumes with $\hat{Q}_{0}=10^{-6}, \Gamma_{0}=1$ and $\gamma=0,1,5,10$.

Figure 6. Variation of: (a) the dimensionless volume flux on log-log scales, (b) momentum flux on log-log scales, (c) buoyancy flux, (d) flux of species A, (e) flux of species B, (f) the local value of $\Gamma,(\mathrm{g})$ the plume width and (h) the centreline velocity when $\varepsilon=1, \gamma=1, \hat{Q}_{0}=$ $10^{-6}, \Gamma_{0}=1$ and $\hat{Q}_{0}=0.1,1,10$. The dotted lines in parts (a) and (b) represent the power-law variations for an inert plume.

Figure 7. Variation of: (a) the dimensionless volume flux on log-log scales, (b) momentum flux on log-log scales, (c) buoyancy flux, (d) flux of species A, (e) flux of species B, (f) the local value of $\Gamma,(\mathrm{g})$ the plume width and (h) the centreline velocity, when $\hat{Q}_{0}=\varepsilon=\gamma=1$ and $\Gamma_{0}=1,0.5,0.05,0.005$. The dotted lines in parts (a) and (b) represent the power-law variations for an inert plume. 
Figure 8. Variation of: (a) the dimensionless volume flux, (b) momentum flux, (c) buoyancy flux, (d) flux of species A, (e) flux of species B, (f) the local value of $\Gamma$, (g) the plume width and (h) the centreline velocity, when $\hat{Q}_{0}=\varepsilon=\lambda=1$ and $\Gamma_{0}=1,2,5,10$.

Figure 9. Variation of: (a) the dimensionless volume flux, (b) momentum flux, (c) buoyancy flux, (d) flux of species A, (e) flux of species B, (f) the local value of $\Gamma$, (g) the plume width and (h) the centreline velocity when $\hat{B}_{r x n}=-1$ and -1.1 . Here, $\varepsilon=-1,-1.1, \gamma=1, \hat{Q}_{0}=10^{-6}$ and $\Gamma_{0}=1$.

Figure 10. Variation of the maximum height of a collapsing plume as a function of $\varepsilon$ for $\gamma=$ $0,1,10$. Here $\hat{Q}_{0}=10^{-6}$ and $\Gamma_{0}=1$. 
Figures
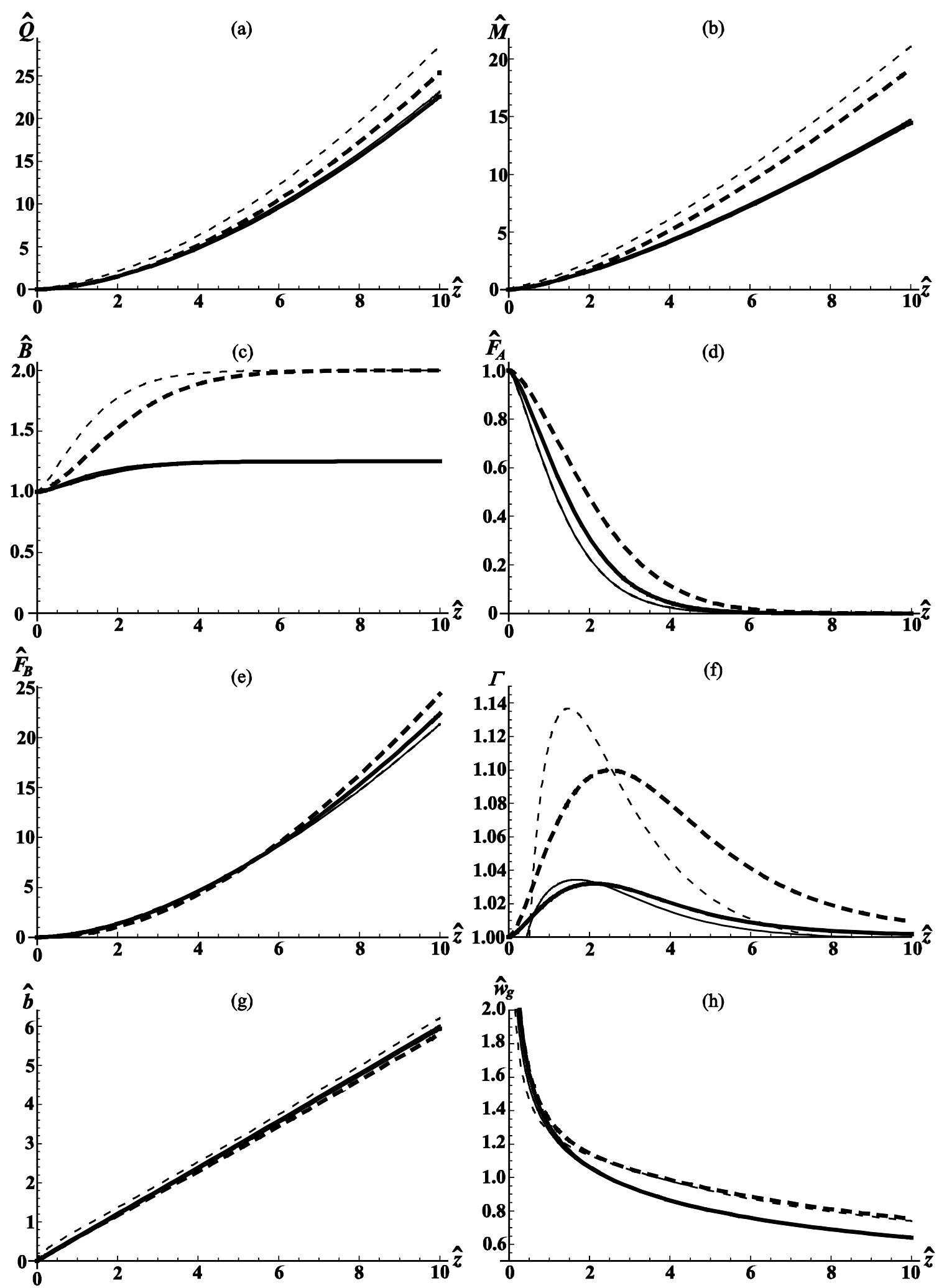

Figure 1 

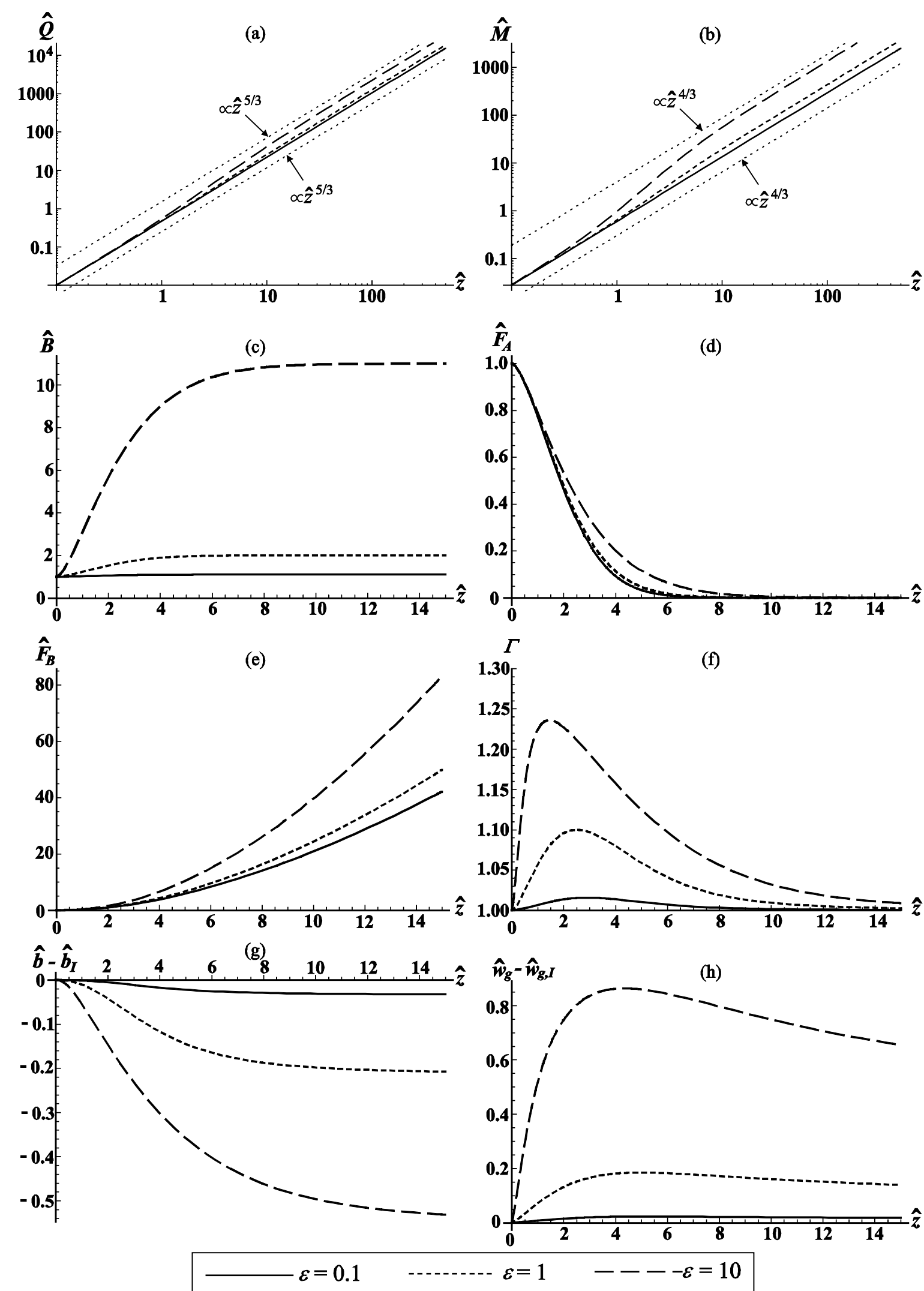

Figure 2 

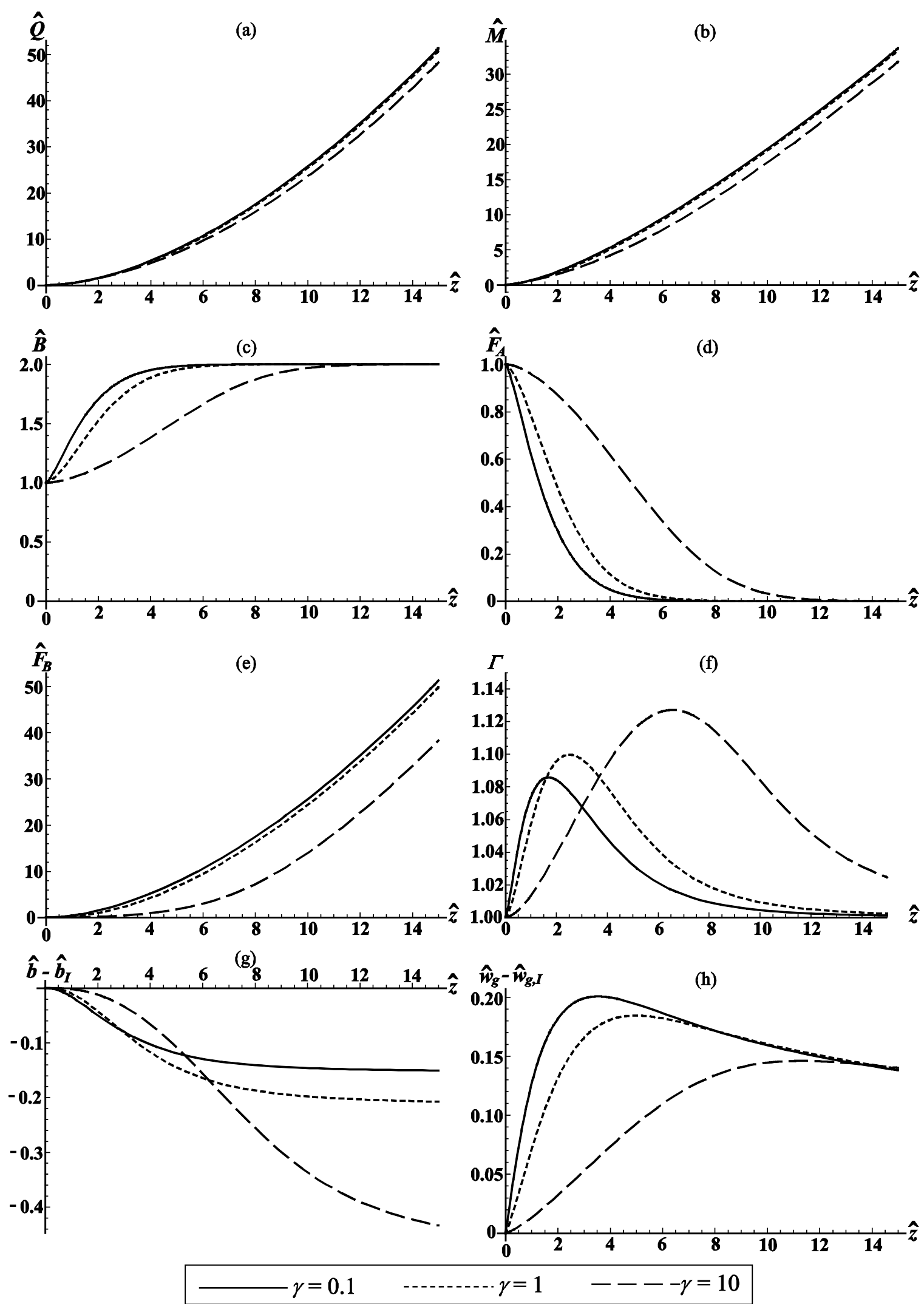

Figure 3 

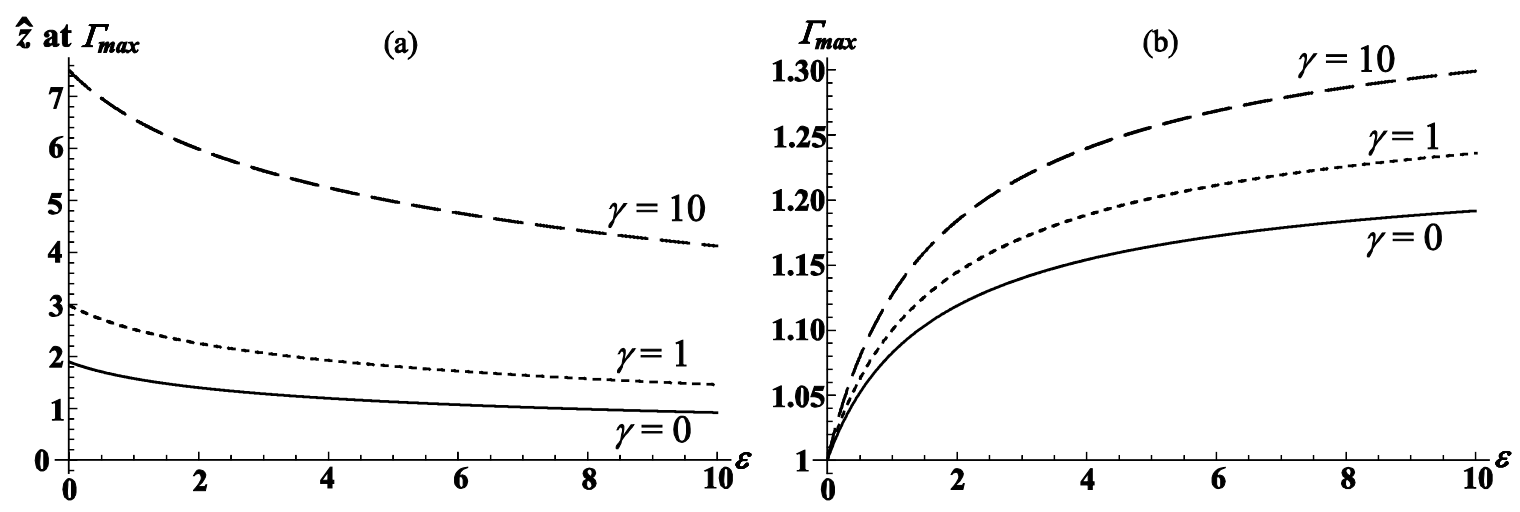

Figure 4

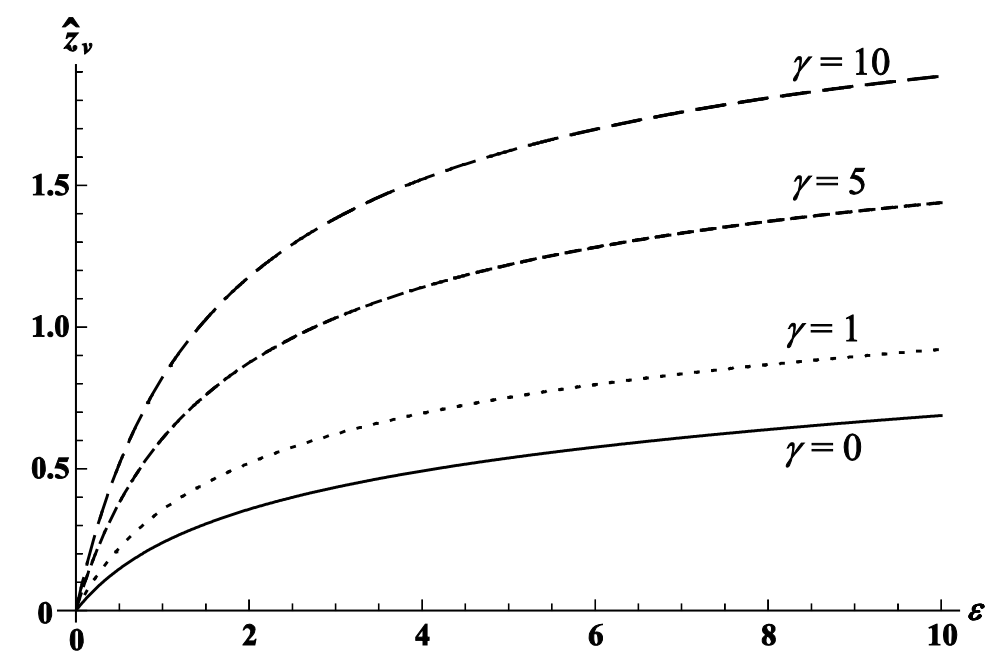

Figure 5 

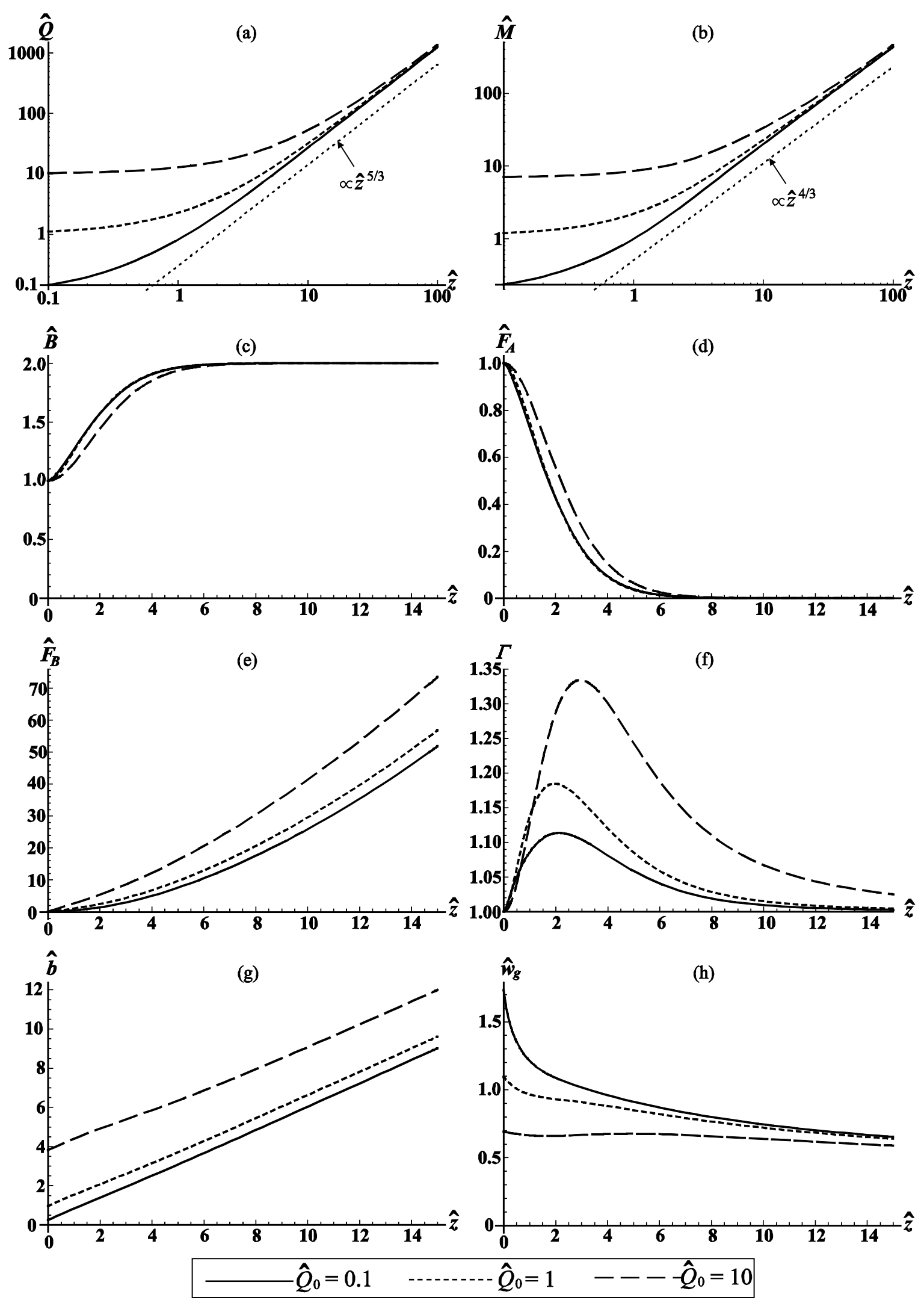

Figure 6 

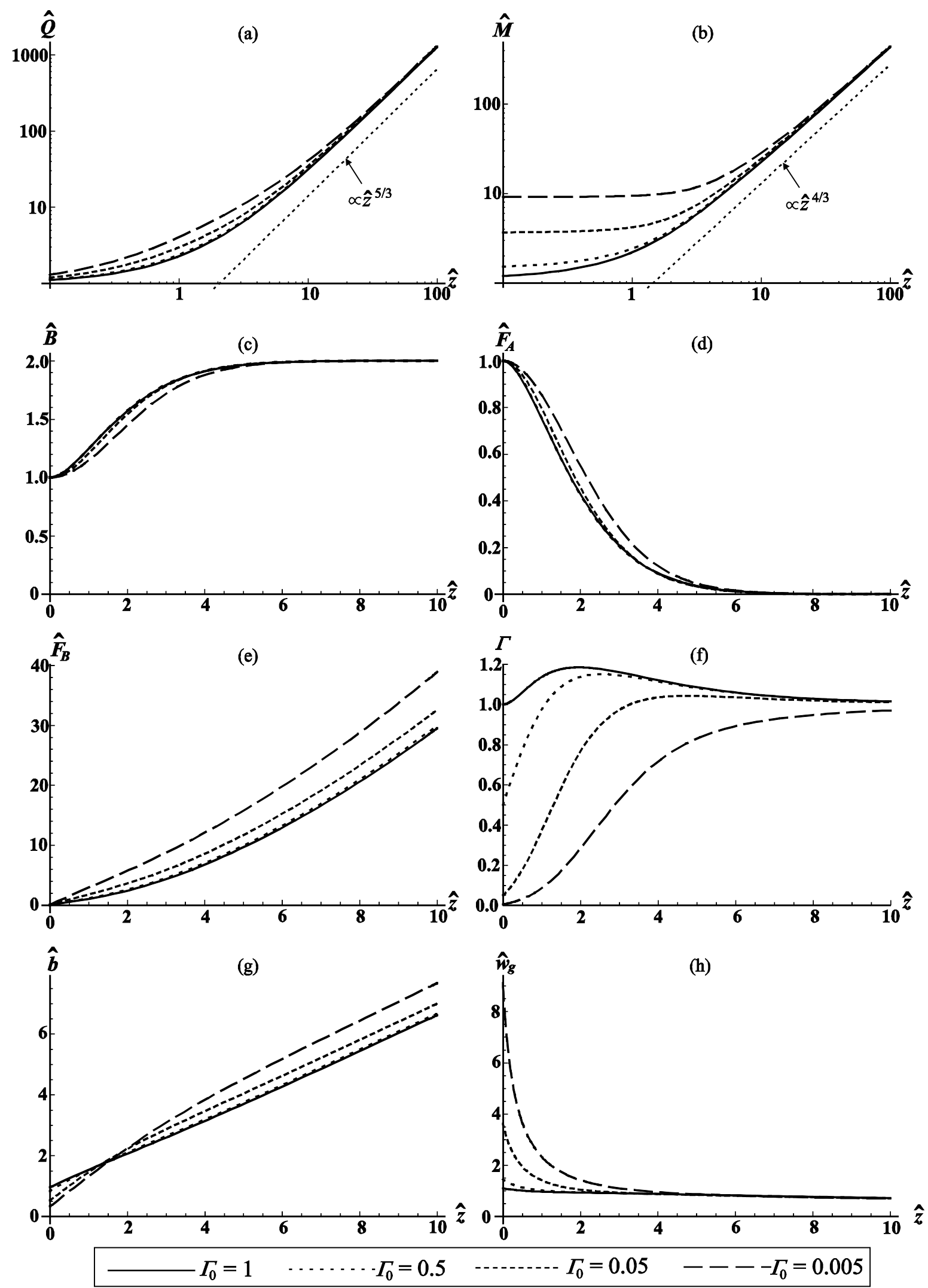

Figure 7 

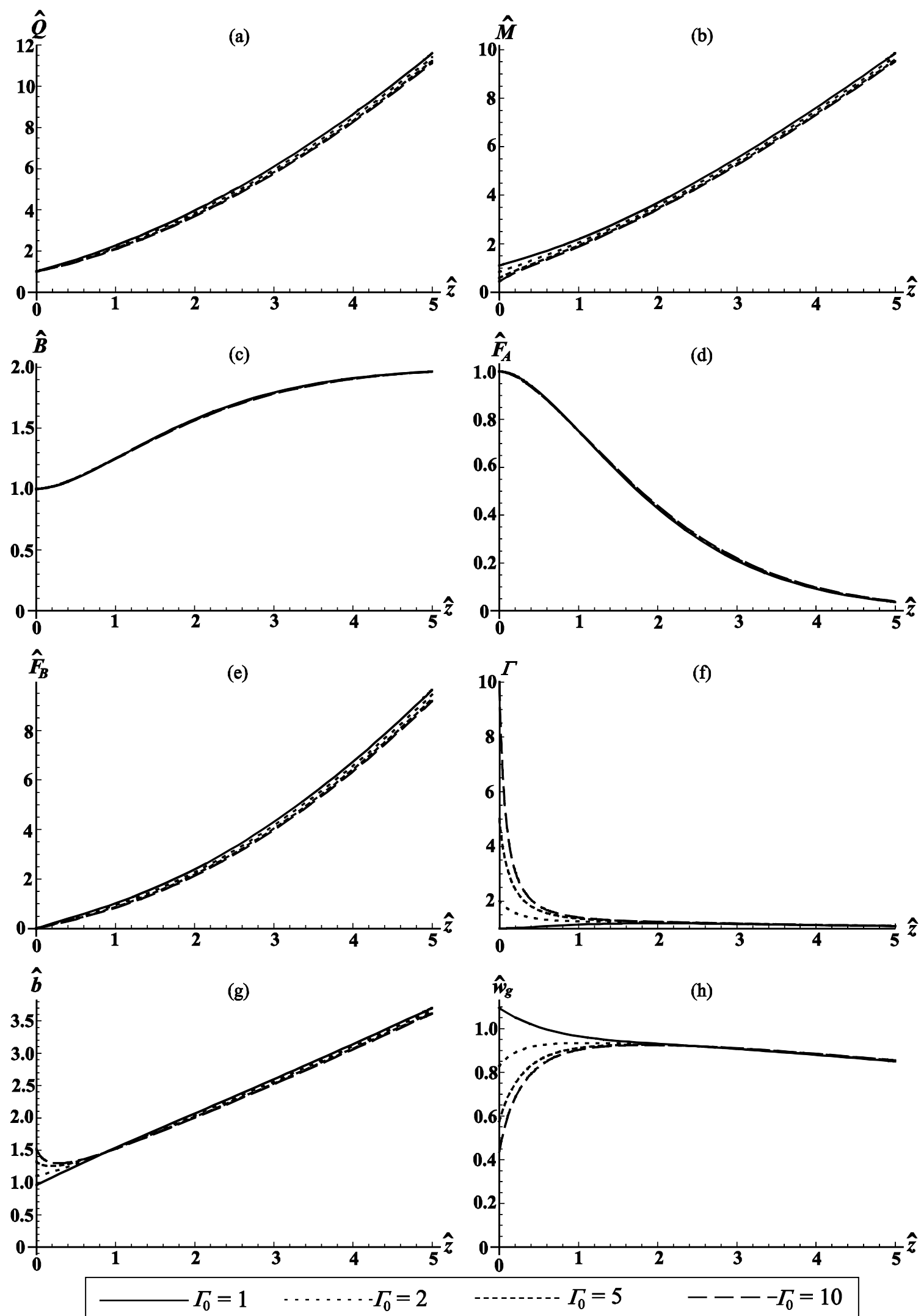

Figure 8 

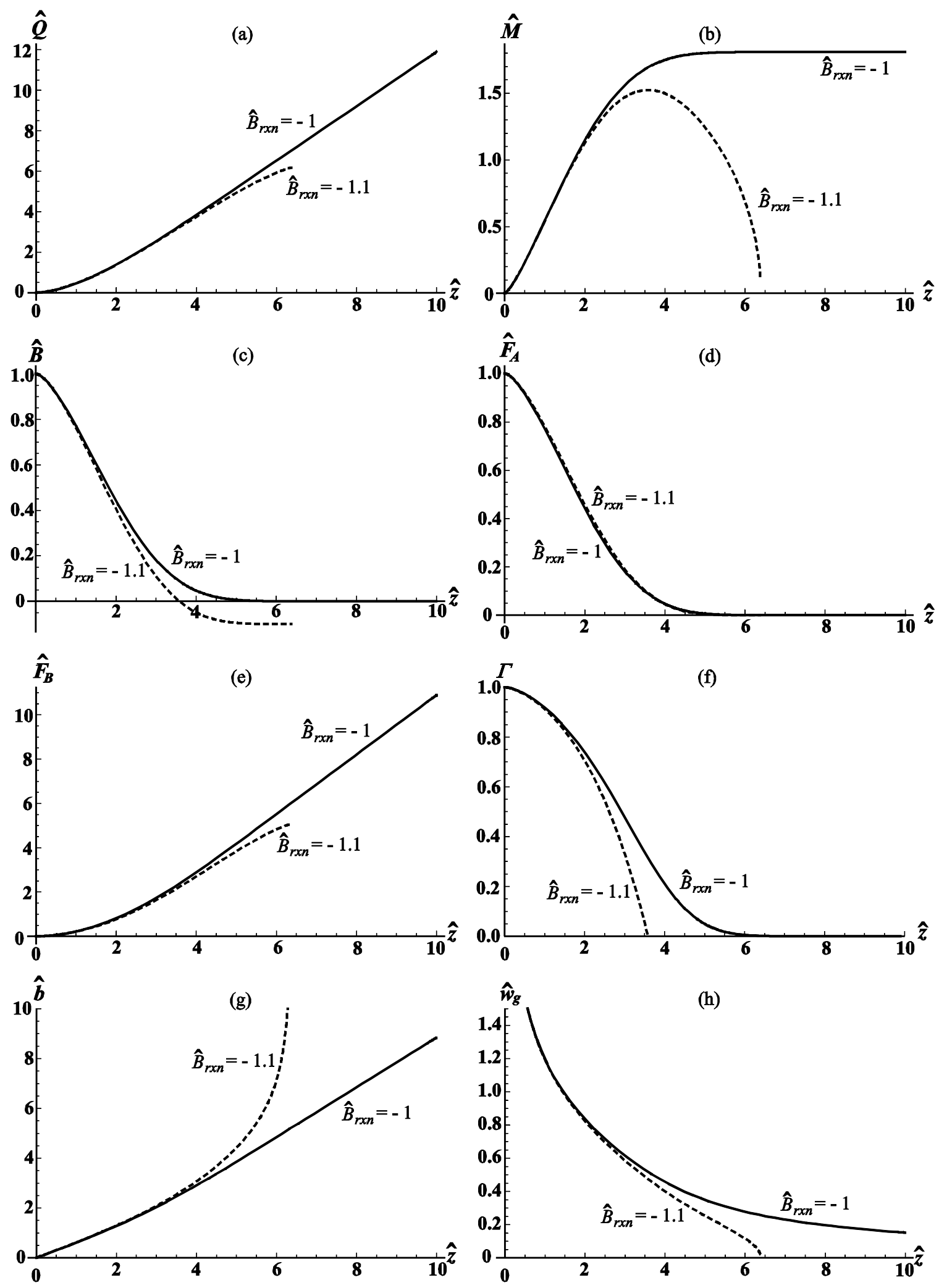

Figure 9 


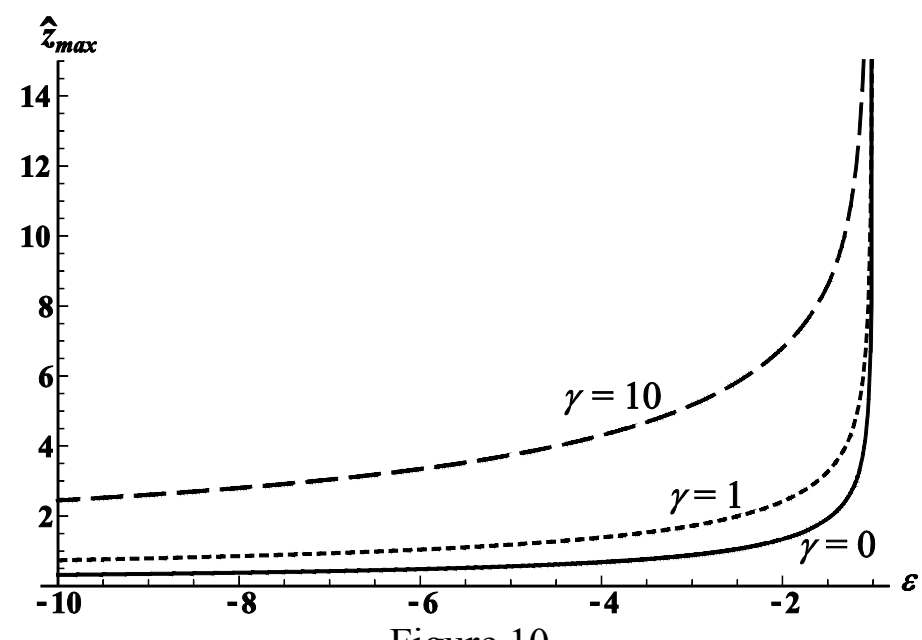

Figure 10 